\title{
GHOST NUMBERS OF GROUP ALGEBRAS
}

\author{
J. DANIEL CHRISTENSEN AND GAOHONG WANG
}

\begin{abstract}
Motivated by Freyd's famous unsolved problem in stable homotopy theory, the generating hypothesis for the stable module category of a finite group is the statement that if a map in the thick subcategory generated by the trivial representation induces the zero map in Tate cohomology, then it is stably trivial. It is known that the generating hypothesis fails for most groups. Generalizing work done for $p$-groups, we define the ghost number of a group algebra, which is a natural number that measures the degree to which the generating hypothesis fails. We describe a close relationship between ghost numbers and Auslander-Reiten triangles, with many results stated for a general projective class in a general triangulated category. We then compute ghost numbers and bounds on ghost numbers for many families of $p$-groups, including abelian $p$-groups, the quaternion group and dihedral 2-groups, and also give a general lower bound in terms of the radical length, the first general lower bound that we are aware of. We conclude with a classification of group algebras of $p$-groups with small ghost number and examples of gaps in the possible ghost numbers of such group algebras.
\end{abstract}

\section{COnTEnts}

1. Introduction

2. The generating hypothesis and the ghost projective class

2.1. The stable module category

2.2. The generating hypothesis

2.3. The ghost projective class

3. Auslander-Reiten triangles and generating lengths

3.1. Relations between the lengths of objects in a triangle

3.2. Auslander-Reiten triangles give composites of ghosts

3.3. Auslander-Reiten triangles, irreducible maps and lengths

3.4. Ghost lengths

4. Ghost numbers of $p$-groups

4.1. Background

4.2. Generating and socle lengths

4.3. Ghost numbers of abelian $p$-groups

4.4. Ghost number of the quaternion group $Q_{8}$

4.5. $\quad$-groups with cyclic normal subgroups

4.6. Ghost numbers of dihedral 2-groups

4.7. Ghost number of $C_{p^{r}} \times C_{p^{s}}$

4.8. Possible ghost numbers for group algebras

References

Department of Mathematics, University of Western Ontario, London, On N6A 5B7, Canada

E-mail addresses: jdc@uwo.ca, gwang72@uwo.ca.

Date: June 27, 2014.

2010 Mathematics Subject Classification. Primary 20C20; Secondary 16G70, 18E30, 20J06, 55P99.

Key words and phrases. Tate cohomology, stable module category, p-group, generating hypothesis, ghost map. 


\section{IntRoduction}

In modular representation theory, the Tate cohomology functor plays a central role, analogous to the role that the homotopy groups play in homotopy theory. Thus it is natural to study the kernel of Tate cohomology, that is, the collection of maps which induce the zero map in Tate cohomology. These maps are called ghosts, and are the topic of the present paper.

Let $G$ be a finite group, and let $k$ be a field whose characteristic $p$ divides the order of $G$. We write $\operatorname{StMod}(k G)$ for the stable module category of $k G$, the triangulated category formed from the module category by killing the projectives, $\operatorname{stmod}(k G)$ for the full subcategory of finitely generated modules, and Thick $\langle k\rangle$ for the thick subcategory generated by the trivial representation, a full subcategory of $\operatorname{stmod}(k G)$. (See Section 2 for complete definitions and further background.)

The generating hypothesis ( $\mathrm{GH}$ ) for the stable module category is the statement that if a map in Thick $\langle k\rangle$ induces the zero map in Tate cohomology, then it is stably trivial. Using the terminology of the first paragraph, this is equivalent to saying that all ghosts in Thick $\langle k\rangle$ are trivial. This problem is motivated by Freyd's famous conjecture in homotopy theory [13], which is still open.

By work of Benson, Carlson, Chebolu, Christensen and Mináč (Theorem 2.1 below), it is known that the generating hypothesis fails for most groups. The extent to which it fails is measured by the ghost number of $k G$, which is the smallest number $n$ such that every composite of $n$ ghosts in Thick $\langle k\rangle$ is stably trivial. With this terminology, the generating hypothesis is the statement that the ghost number is one. The ghost number was studied for $p$-groups in [9], but even for $p$-groups it was found to be difficult to calculate, and in most cases only crude bounds are known. It is a long-term goal to understand whether this invariant has a simple description in terms of other invariants of $k G$.

In the present paper we develop new techniques for the study of ghost numbers and use them to make new computations in many cases. For example, we make the first computations of the ghost numbers of group algebras of wild representation type at an odd prime $\left(k\left(C_{3} \times C_{3}\right)\right.$ and others mentioned in the detailed summary below) as well as the first computations of the ghost numbers of non-abelian group algebras (the dihedral 2-groups).

Theorem 4.28. Let $G=C_{3} \times C_{3}$, and let $k$ be a field of characteristic 3 . Then the ghost number of $k G$ is 3 .

Corollary 4.25. Let $k$ be a field of characteristic 2. Then the ghost number of $k D_{4 q}$ is $q+1$ for all $q \geqslant 1$, where $D_{4 q}$ denotes the dihedral 2-group of order $4 q$, with $q$ a power of 2 .

Our followup paper [12] builds on the work here in order to compute the ghost numbers of non- $p$ groups. For example, using Corollary 4.25 , we are able to compute the ghost number of an arbitrary dihedral group at the prime 2 in 12$]$.

We also give many new bounds on ghost numbers, including lower bounds, which are generally difficult to come by. We highlight two such results here:

Corollary 4.17. Let $G$ be a p-group, and let $k$ be a field of characteristic $p$. Then

$$
\frac{1}{2} \text { radlen } k G \leqslant \text { ghost num } k G \leqslant \text { gen num } k G<\operatorname{rad} \text { len } k G,
$$

when $p$ is even, and

$$
\frac{1}{3} \text { radlen } k G \leqslant \text { ghost num } k G \leqslant \text { gen num } k G<\operatorname{rad} \text { len } k G,
$$

when $p$ is odd.

Proposition 4.33. Let $k$ be a field of characteristic $p$. If $G$ is a group of order $p^{r}$, then the ghost number of $k G$ is at least $(r-1)(p-1)+1$.

Our work also includes results which are quite general, in some cases applying to any projective class in any triangulated category. 
We now give a detailed summary of the contents of the paper. We begin in Section 2.1 by reviewing the stable module category. In Section 2.2 we recall the statement of the generating hypothesis in this situation and state the result of Benson, Carlson, Chebolu, Christensen and Minác that says that the GH fails unless the Sylow $p$-subgroup of $G$ is $C_{2}$ or $C_{3}$. The ghost number, which measures the degree to which the GH fails, is best studied using the idea of a projective class, so we introduce projective classes and their associated invariants in Section 2.3. Briefly, a projective class consists of a collection $\mathcal{P}$ of objects (thought of as "projective" building blocks) and an ideal $\mathcal{I}$ of morphisms (the maps invisible to the objects in $\mathcal{P}$ ) satisfying some axioms. In this section, we also define the invariants we will study. The ghost length of a $k G$-module $M$ is the smallest number $n$ such that every composite of $n$ ghosts in Thick $\langle k\rangle$ starting from $M$ is stably trivial. The ghost number that we introduced previously is the supremum of the ghost lengths of modules in Thick $\langle k\rangle$. In addition, it is convenient to define the generating length of $M$ to be the smallest number $n$ such that every composite of $n$ ghosts in $\operatorname{StMod}(k G)$ starting from $M$ is trivial, and the generating number of $k G$ to be the supremum of the generating lengths of modules in Thick $\langle k\rangle$.

In Section 3 we present a variety of new results, many of which hold for arbitrary (stable) projective classes in arbitrary triangulated categories. For example, in Section 3.1, we give new bounds on the length of an object in a triangle in terms of the lengths of the other two objects and the filtration of the connecting homomorphism in the powers of the ideal. Then, in Section 3.2, we show that the connecting map $\gamma: Z \rightarrow \Sigma X$ in an Auslander-Reiten triangle, which we call the almost zero map, has a remarkable property: if $(\mathcal{P}, \mathcal{I})$ is any projective class such that there is a nonzero map from $Z$ in $\mathcal{I}^{k}$, then $\gamma$ is in $\mathcal{I}^{k}$. So the almost zero map is in some sense a universal example of a non-zero map from $Z$. We specialize to the case of the stable module category in Section 3.3, where we show that the heart of an indecomposable module $M$ (the fibre of the almost zero map) has length which differs by at most one from $M$, with respect to any projective class. We also show that this is true for any summand of the heart, by showing that the lengths of the domain and codomain of any irreducible map differ by at most one. We finish Section 3 with Section 3.4, which describes the extent to which our results hold for the ghost length, the invariant used in defining the ghost number.

Section 4 contains detailed computational results on the ghost numbers of $p$-groups. We begin by recalling some background results in Section 1.1, such as the fact that the ghost number of $k G$ is less than the nilpotency index of the Jacobson radical, as well as the fact that multiplication by $x-1$, where $x$ is a central element of $G$, is always a ghost. In Section 4.2 we show that the generating length invariant is in a precise sense a stabilized version of the socle length, and show that if these are equal for a module $M$, the same is true for $\operatorname{rad}(M)$ and $M / \operatorname{soc}(M)$. This follows from a general result involving nested unstable projective classes in a triangulated category. We begin our computations in Section 4.3, where we study the ghost numbers of abelian $p$-groups. The main result here is an improved lower bound on the ghost number. This follows from a result giving a lower bound on the ghost length of induced modules for general $p$-groups. We also compute the exact ghost length of many modules over abelian $p$-groups. In Section 4.4 we show that the ghost number for the quaternion group $Q_{8}$ is 3 or 4, improving the existing lower bound by 1. In Section 1.5, we compute the ghost length and generating length of certain modules induced up from a cyclic normal subgroup of a $p$-group, generalizing the technique used for $Q_{8}$. This is used in the same section to show that the ghost number and the radical length are within a factor of three of each other for any $p$-group. More precisely, we show that $(\operatorname{rad}$ len $k G) / 3 \leqslant$ ghost num $k G<\operatorname{rad}$ len $k G$ for $p$ odd, the first general lower bound we are aware of. For $p=2$, the factor of 3 is replaced with a factor of 2 . We also use the induction result in Section 4.6, where we show that the ghost number of the dihedral 2-group $D_{4 q}$ of order $4 q$ is exactly $q+1$. This is the longest section of the paper. That the ghost length is at least $q+1$ follows immediately from the induction result of the previous section, but that it is no more than $q+1$ requires using the classification of $k D_{4 q}$-modules. In Section 4.7 we show that the ghost number of $k\left(C_{3} \times C_{3}\right)$ is exactly 3 . While $k\left(C_{3} \times C_{3}\right)$-modules are not classifiable, we make use of the fact that certain quotients can be classified. Our argument also shows that the 
ghost number of the group algebra $k\left(C_{p^{r}} \times C_{p^{s}}\right)$, for $p^{r}, p^{s}>2$, is at most $p^{r}+p^{s}-3$. It follows that the ghost number of $k\left(C_{3} \times C_{3^{s}}\right)$ is $3^{s}$ and that the ghost number of $k\left(C_{4} \times C_{2^{s}}\right)$ is $2^{s}+1$. We end the paper with Section 4.8, in which we give complete lists of the group algebras of $p$-groups with ghost numbers 1,2 or 3 , with the possible exception of $k Q_{8}$. We also prove that for each prime $p$ there are gaps in the possible ghost numbers that can occur, and state a conjecture related to this.

Our work also raises various questions, which we briefly summarize here. We have shown that the generating number is a stabilized version of the radical length, and that both the generating number and ghost number are within a constant factor of the radical length, but it still remains to fully understand these new invariants and determine whether they have an exact description in terms of existing invariants.

Question 1.1. How do the ghost number and generating number relate to other invariants of the group algebra?

Moreover, in all examples where they have been computed, the ghost number and the generating number agree, and we conjecture that this is always the case.

Conjecture 1.2. Let $G$ be a finite group, and let $k$ be a field whose characteristic divides the order of $G$. Then the ghost number of $k G$ is equal to the generating number of $k G$.

We also believe that the ghost number of a general $p$-group is bounded in the following way, as described in Section 4.8 .

Conjecture 1.3. Let $k$ be a field of characteristic $p$. If $G$ is a p-group of order $p^{r}$, then

ghost number of $k\left(C_{p}^{r}\right) \leqslant$ ghost number of $k G \leqslant$ ghost number of $k\left(C_{p^{r}}\right)$.

\section{The GENERATING HYPOTHESIS AND THE GHOST PROJECTIVE CLASS}

In this section, we recall background material which provides context to our results and which we use in our proofs.

\subsection{The stable module category}

Here we recall the basics of the stable module category. A good reference is $[6]$.

Let $G$ be a finite group, and let $k$ be a field whose characteristic $p$ divides the order of $G$. The stable module category $\operatorname{StMod}(k G)$ is a quotient category of the category $\operatorname{Mod}(k G)$ of left $k G-$ modules by the ideal of maps that factor through a projective. Thus the objects of $\operatorname{StMod}(k G)$ are left $k G$-modules and the hom-sets are $\underline{\operatorname{Hom}}(M, N)=[M, N]:=\operatorname{Hom}(M, N) / \operatorname{PHom}(M, N)$, where $\operatorname{PHom}(M, N)$ denotes the stably trivial maps, i.e., those that factor through a projective module. Two modules $M$ and $N$ are isomorphic in the stable module category if and only if they have the same projective-free summands. In particular, projective modules are isomorphic to zero in the stable module category. We write $\operatorname{stmod}(k G)$ for the full subcategory of finitely generated $k G$-modules in $\operatorname{StMod}(k G)$. (More precisely, we include all modules which are stably isomorphic to finitely generated $k G$-modules.)

The stable module category is a triangulated category. The desuspension $\Omega M$ of a module $M$ is the kernel of any surjection $P \rightarrow M$ with $P$ projective. This is well-defined in the stable module category by Schanuel's Lemma [6, Prop. 4.2], and we write $\tilde{\Omega} M$ for the projective-free summand of $\Omega M$.

The group algebra $k G$ is injective as a module over itself. In particular, this implies that projective modules and injective modules coincide in $\bmod (k G)$. The suspension $\Sigma N$ of a module $N$ is defined to be the cokernel of any injection $N \rightarrow P$ with $P$ injective. We will often write $\Omega^{-1} N$ for $\Sigma N$ since $\Omega$ and $\Sigma$ are inverse functors up to natural isomorphism.

Write $k$ for the trivial representation and Thick $\langle k\rangle$ for the thick subcategory generated by $k$, the smallest full triangulated subcategory of $\operatorname{StMod}(k G)$ that is closed under retracts and contains $k$. This is in fact a full subcategory of $\operatorname{stmod}(k G)$, and plays a central role in our formulation of the 
generating hypothesis. The localizing category generated by $k$, denoted $\operatorname{Loc}\langle k\rangle$, is the smallest full triangulated subcategory of $\operatorname{StMod}(k G)$ that is closed under arbitrary coproducts and retracts and contains $k$.

\subsection{The generating hypothesis}

An important feature of the stable module category is that the Tate cohomology of a $k G$-module $M$ is representable, i.e., we have a canonical isomorphism $\hat{H}^{n}(G, M) \cong\left[\Omega^{n} k, M\right]$.

We say that the generating hypothesis $(\mathbf{G H})$ holds for the stable module category $\operatorname{StMod}(k G)$ if and only if the Tate cohomology functor $\hat{H}^{*}(G,-)$ restricted to Thick $\langle k\rangle$ is faithful. It has been shown that the GH fails for most group algebras [3, 6, 8, 10].

Theorem 2.1 (Benson, Carlson, Chebolu, Christensen and Mináč). Let $G$ be a finite group, and let $k$ be a field whose characteristic $p$ divides the order of $G$. Then the $G H$ holds for $\operatorname{StMod}(k G)$ if and only if the Sylow p-subgroup $P$ of $G$ is either $C_{2}$ or $C_{3}$.

It is worth pointing out here why we restrict to Thick $\langle k\rangle$. It is known that whenever the thick subcategory is not all of $\operatorname{stmod}(k G)$, there are non-projective modules whose Tate cohomology is zero. The identity map on such a module is sent to zero by $\hat{H}^{*}(G,-)$, so the GH would be trivially false if we included such modules. Restricting to Thick $\langle k\rangle$ prevents this from happening. In general, the stable module category is generated by the simple modules as a triangulated category. For a $p$-group $G$, the trivial representation $k$ is the only simple module, so we have that $\operatorname{Thick}\langle k\rangle=\operatorname{stmod}(k G)$ in this case.

We call a map in $\operatorname{StMod}(k G)$ that is in the kernel of the Tate cohomology functor a ghost. Thus the GH is the statement that all ghosts in Thick $\langle k\rangle$ are stably trivial. When the GH fails, the vanishing of composites of ghosts gives a measure of the failure and leads to invariants of modules and of $k G$. This is formalized in the idea of a projective class.

\subsection{The ghost projective class}

In this section, we introduce the invariants that are the subject of this paper: generating length, ghost length, generating number and ghost number. These are defined using the concept of a projective class:

Definition 2.2. Let $\mathbb{T}$ be a triangulated category. A projective class in $\mathbb{T}$ consists of a class $\mathcal{P}$ of objects of $\mathbb{T}$ and a class $\mathcal{I}$ of morphisms of $\mathbb{T}$ such that:

(i) $\mathcal{P}$ consists of exactly the objects $P$ such that every composite $P \rightarrow X \rightarrow Y$ is zero for each $X \rightarrow Y$ in $\mathcal{I}$,

(ii) $\mathcal{I}$ consists of exactly the maps $X \rightarrow Y$ such that every composite $P \rightarrow X \rightarrow Y$ is zero for each $P$ in $\mathcal{P}$.

(iii) for each $X$ in $\mathbb{T}$, there is a cofibre sequence $P \rightarrow X \rightarrow Y$ with $P$ in $\mathcal{P}$ and $X \rightarrow Y$ in $\mathcal{I}$.

In this paper, we make the additional assumption that the projective class is stable, that is, that $\mathcal{P}$ (or equivalently $\mathcal{I}$ ) is closed under suspension and desuspension. With slight alterations, most of our results remain true without this assumption, but the extra bookkeeping complicates the arguments. The one exception is that in Section 4.2 we make use of an unstable projective class.

Remark 2.3. It follows from the definition that $\mathcal{P}$ is closed under arbitrary coproducts and retracts, and that $\mathcal{I}$ is an ideal.

We write $\mathcal{G}$ for the ideal of ghosts in the stable module category, and $\mathcal{F}$ for all retracts of direct sums of suspensions of $k$ in $\operatorname{StMod}(k G)$. For a module $M \in \operatorname{StMod}(k G)$, since $\hat{H}^{n}(G, M) \cong\left[\Omega^{n} k, M\right]$, we can form a map $\oplus \Omega^{i} k \rightarrow M$ that is surjective on Tate cohomology by assembling sufficiently many homogeneous elements in $\hat{H}^{*}(G, M)$. Completing this map into a triangle in $\operatorname{StMod}(k G)$

$$
\Omega U_{M} \rightarrow \oplus \Omega^{i} k \rightarrow M \stackrel{\phi_{M}}{\longrightarrow} U_{M},
$$


we get a ghost $\phi_{M}: M \rightarrow U_{M}$. The map $\phi_{M}$ is a (weakly) universal ghost in the sense that every ghost out of $M$ factors though it, but the factorization is not necessarily unique. It follows easily that $(\mathcal{F}, \mathcal{G})$ forms a projective class in $\operatorname{StMod}(k G)$. This is called the ghost projective class.

While the ghost projective class is the focus of this paper, some of our results apply to any projective class, so we mention two other examples at this point: The simple ghost projective class is the projective class whose projectives are generated by all simple objects, and it was proposed for study in [5] as a way to avoid focusing on Thick $\langle k\rangle$. And the strong ghost projective class is the projective class whose ideal consists of the maps which are ghosts under restriction to every subgroup. The last example has been studied by Carlson, Chebolu and Mináč in work in progress, and all three of the projective classes defined above are studied in [12].

For any projective class $(\mathcal{P}, \mathcal{I})$, there is a sequence of derived projective classes $\left(\mathcal{P}_{n}, \mathcal{I}^{n}\right)[11$. The ideal $\mathcal{I}^{n}$ consists of all $n$-fold composites of maps in $\mathcal{I}$, and $X$ is in $\mathcal{P}_{n}$ if and only if it is a retract of an object $M$ that sits inside a cofibre sequence $P \rightarrow M \rightarrow Q$ with $P \in \mathcal{P}_{1}=\mathcal{P}$ and $Q \in \mathcal{P}_{n-1}$. For $n=0$, we let $\mathcal{P}_{0}$ consist of all zero objects and $\mathcal{I}^{0}$ consist of all maps in $\mathbb{T}$. The length $\operatorname{len}_{\mathcal{P}}(X)$ of an object $X$ of $\mathbb{T}$ with respect to $(\mathcal{P}, \mathcal{I})$ is the smallest $n$ such that $X$ is in $\mathcal{P}_{n}$, if this exists. The fact that each pair $\left(\mathcal{P}_{n}, \mathcal{I}^{n}\right)$ is a projective class implies that the length of $X$ is equal to the smallest $n$ such that every map in $\mathcal{I}^{n}$ with domain $X$ is trivial.

The length of a module $M$ with respect to the ghost projective class is called the generating length of $M$, and this exists when $M$ is in Thick $\langle k\rangle$. But since we are interested in the collection $\mathcal{G}_{t}$ of ghosts in Thick $\langle k\rangle$, we also get another invariant. We describe both invariants, and the associated invariants of $k G$, in the following definition, generalizing the definition given in [9] for $p$-groups.

\section{Definition 2.4.}

- The generating length $\operatorname{gel}(M)$ of $M \in \operatorname{Thick}\langle k\rangle$ is the smallest $n$ such that $M \in \mathcal{F}_{n}$. That is, $\operatorname{gel}(M)=\operatorname{len}_{\mathcal{F}}(M)$.

- The ghost length $\operatorname{gl}(M)$ of $M \in$ Thick $\langle k\rangle$ is the smallest integer $n$ such that every map in $\left(\mathcal{G}_{t}\right)^{n}$ with domain $M$ is trivial.

- The generating number of $k G$ is the least upper bound of the generating lengths of modules in Thick $\langle k\rangle$.

- The ghost number of $k G$ is the least upper bound of the ghost lengths of modules in Thick $\langle k\rangle$.

With this terminology, the generating hypothesis is the statement that the ghost number of $k G$ is 1 .

Let $M$ be in Thick $\langle k\rangle$. Since each $\left(\mathcal{F}_{n}, \mathcal{G}^{n}\right)$ is a projective class and $\left(\mathcal{G}_{t}\right)^{n} \subseteq \mathcal{G}^{n}$, it follows that

$$
\operatorname{gl}(M) \leqslant \operatorname{gel}(M)
$$

and therefore that

ghost number of $k G \leqslant$ generating number of $k G$.

When $G$ has periodic Tate cohomology, the coproduct in (2.1) can be taken to be finite, and it follows that the ghost projective class restricts to a projective class in Thick $\langle k\rangle$ [9]. This implies that equality holds in this case. We don't know whether equality holds in general, except for the trivial observation that $M \cong 0$ if and only $\operatorname{gel}(M)=0$ if and only if $\operatorname{gl}(M)=0$ and the less trivial fact that $\operatorname{gel}(M)=1$ if and only if $\operatorname{gl}(M)=1$ (see Corollary 3.7 or $[7 \mid$ ). Thus the GH is equivalent to the generating number of $k G$ being 1 . See Remark 3.13 for further discussion of whether ghost length equals generating length.

\section{Auslander-Reiten triangles And generating Lengths}

In this section, we explain how Auslander-Reiten triangles (in short, A-R triangles) provide examples of ghosts, and, more generally, of non-trivial maps in $\mathcal{I}^{n}$ for $n$ as large as possible, for

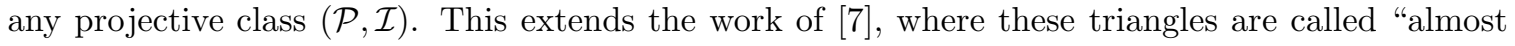


split sequences." Because we have in mind applications to other projective classes, in this section we state many of our results for a general projective class in a general triangulated category.

In Section 3.1, we give results about the relationship between the lengths of the objects in a triangle when one of the maps is in a power $\mathcal{I}^{m}$ of the ideal. In Section 3.2, we recall A-R triangles and prove that the third map in an A-R triangle is the longest possible non-trivial composite of maps in $\mathcal{I}$ with the given domain. In Section 3.3, we apply these results to the study of lengths in the stable module category, and also show a close relationship between lengths and irreducible maps. Finally, in Section 3.4 we explain the extent to which our results on generating length are true for ghost length.

\subsection{Relations between the lengths of objects in a triangle}

Consider a projective class $(\mathcal{P}, \mathcal{I})$ in a triangulated category $\mathbb{T}$. Let

$$
X \stackrel{\alpha}{\rightarrow} Y \stackrel{\beta}{\rightarrow} Z \stackrel{\gamma}{\rightarrow} \Sigma X
$$

be a triangle in $\mathbb{T}$, where $X, Y$ and $Z$ have finite lengths $k, n$ and $l$, respectively. We know that $n \leqslant k+l[11$. Rotating the triangle, we also get $l \leqslant n+k$ and $k \leqslant n+l$. Here we show that when $\gamma$ is in $\mathcal{I}^{m}$, one can refine these inequalities by subtracting $m$ from $l$. Our methods also show that $n \geqslant m$. Note that $\mathcal{I}^{0}$ consists of all maps in $\mathbb{T}$.

Lemma 3.1. Let $(\mathcal{P}, \mathcal{I})$ be a projective class in a triangulated category $\mathbb{T}$, and let

$$
X \stackrel{\alpha}{\rightarrow} Y \stackrel{\beta}{\rightarrow} Z \stackrel{\gamma}{\rightarrow} \Sigma X
$$

be a triangle in $\mathbb{T}$, where $X, Y$ and $Z$ have finite lengths $k, n$ and $l$, respectively, and $\gamma \in \mathcal{I}^{m}$ with $m \leqslant l$. Then

$$
\operatorname{len}_{\mathcal{P}}(Y)=n \leqslant \max (k-m+l, l) .
$$

Note that if $m \geqslant l$, then $\gamma$ must be zero, and so the restriction to $m \leqslant l$ is natural. When $m=l$, the triangle splits, and the lemma says that $n \leqslant \max (k, l)$.

Proof. Let $n^{\prime}=\max (k, m)$, and let $\phi: Y \rightarrow W$ be in $\mathcal{I}^{n^{\prime}}$. Then $\phi \circ \alpha$ is zero (since $n^{\prime} \geqslant k$ ), so $\phi$ factors through a map $\tilde{\phi}: Z \rightarrow W$. We claim that $\tilde{\phi}$ is in $\mathcal{I}^{m}$. Consider the diagram

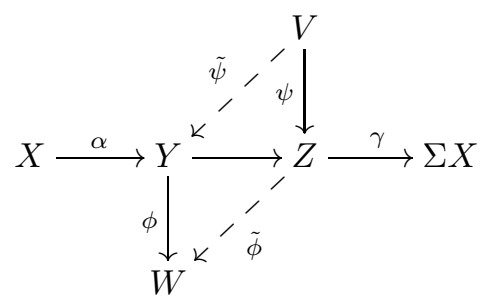

with $\psi: V \rightarrow Z$ being any map from an object $V \in \mathcal{P}_{m}$. Now $\gamma \in \mathcal{I}^{m}$, so $\gamma \circ \psi$ is zero, and $\psi$ factors through some map $\tilde{\psi}: V \rightarrow Y$. Hence $\tilde{\phi} \circ \psi=\phi \circ \tilde{\psi}$ is zero (since $n^{\prime} \geqslant m$ ), and the claim follows. If $g: W \rightarrow W^{\prime}$ is in $\mathcal{I}^{l-m}$, then $g \circ \tilde{\phi}$ is zero because $Z$ has length $l$. Then $g \circ \phi$ is zero, meaning that the length of $Y$ is at most $n^{\prime}+l-m$.

Lemma 3.2. Let $(\mathcal{P}, \mathcal{I})$ be a projective class in a triangulated category $\mathbb{T}$, and let

$$
X \stackrel{\alpha}{\rightarrow} Y \stackrel{\beta}{\rightarrow} Z \stackrel{\gamma}{\rightarrow} \Sigma X
$$

be a triangle in $\mathbb{T}$, where $X, Y$ and $Z$ have finite lengths $k, n$ and $l$, respectively, and $\gamma \in \mathcal{I}^{m}$ with $m \leqslant l$. Then

$$
\operatorname{len}_{\mathcal{P}}(Y)=n \geqslant \max (k-l+m, m) .
$$

When $m=l$, this says that $n \geqslant \max (k, l)$, so the two lemmas together recover the fact that when the triangle splits, $n=\max (k, l)$. 
Proof. We prove that the length of $Y$ is at least $k-l+m$. The other inequality can be proved similarly.

Consider a map $\phi: X \rightarrow W$ in $\mathcal{I}^{l-m}$. Since $\phi \circ \Sigma^{-1} \gamma$ is in $\mathcal{I}^{l}$ and has domain $\Sigma^{-1} Z$ of length $l$, it is zero and $\phi$ factors through a map $\tilde{\phi}: Y \rightarrow W$ :

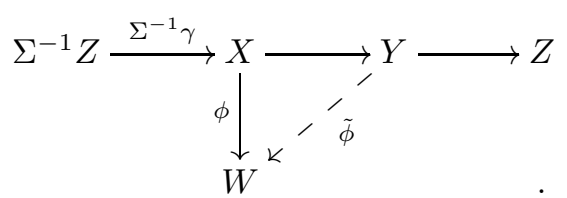

Let $g: W \rightarrow W^{\prime}$ be in $\mathcal{I}^{n}$. Then $g \circ \tilde{\phi}$ is zero because $Y$ has length $n$, hence any map in $\mathcal{I}^{n+l-m}$ with domain $X$ is zero. This implies that $k \leqslant n+l-m$, i.e., that $n \geqslant k-l+m$.

\subsection{Auslander-Reiten triangles give composites of ghosts}

We begin by recalling the definition.

Definition 3.3. Let $\mathbb{T}$ be a triangulated category. A triangle $X \stackrel{\alpha}{\rightarrow} Y \stackrel{\beta}{\rightarrow} Z \stackrel{\gamma}{\rightarrow} \Sigma X$ is called an Auslander-Reiten triangle, if

(a) $\gamma \neq 0$

(b) any map $X \rightarrow Y^{\prime}$ that is not split monic factors through $\alpha$,

(c) any map $Y^{\prime} \rightarrow Z$ that is not split epic factors through $\beta$.

A map $\alpha$ that is not split monic and satisfies (b) is said to be left almost split. Dually, a map $\beta$ that is not split epic and satisfies (C) is said to be right almost split.

We know that Auslander-Reiten triangles exist in great generality.

Theorem 3.4 (Krause, 15]). Let $\mathbb{T}$ be a triangulated category with all small coproducts, and suppose that all cohomological functors are representable. Let $Z$ be a compact object in $\mathbb{T}$ with local endomorphism ring. Then there exists an Auslander-Reiten triangle

$$
X \stackrel{\alpha}{\rightarrow} Y \stackrel{\beta}{\rightarrow} Z \stackrel{\gamma}{\rightarrow} \Sigma X .
$$

The triangle is unique up to a non-canonical isomorphism.

Remark 3.5. Let $\beta$ be the second map in the A-R triangle above. One can show that, for any endomorphism $g$ of $Y$ with $\beta g=\beta$, the map $g$ is an isomorphism (see [15]). We say that the map $\beta$ is right minimal in this case. Dually, the first map $\alpha$ in an A-R triangle is left minimal. A map $\beta$ that is right almost split sits inside an Auslander-Reiten triangle if and only if it is right minimal [15].

For convenience, we call the map $\gamma$ here the almost zero map with domain $Z$. It is unique up to an automorphism of $\Sigma X$. The following proposition follows from the definitions and the earlier lemmas.

Proposition 3.6. Suppose that $(\mathcal{P}, \mathcal{I})$ is a projective class on a triangulated category $\mathbb{T}$, and that

$$
X \stackrel{\alpha}{\rightarrow} Y \stackrel{\beta}{\rightarrow} Z \stackrel{\gamma}{\rightarrow} \Sigma X
$$

is a distinguished triangle with $\beta$ right almost split. If $Z$ has finite length $l$ and $X$ has finite length $k$ with respect to $(\mathcal{P}, \mathcal{I})$, then the third map $\gamma$ is in $\mathcal{I}^{l-1}$, and

$$
\begin{aligned}
& k-1 \leqslant \operatorname{len}_{\mathcal{P}}(Y) \leqslant k+1, \text { if } k \geqslant l ; \\
& l-1 \leqslant \operatorname{len}_{\mathcal{P}}(Y) \leqslant l, \quad \text { if } k \leqslant l-1 .
\end{aligned}
$$

For any summand $S$ of $Y, \operatorname{len}_{\mathcal{P}}(S) \leqslant \max (k+1, l)$. 
Proof. We test $\gamma$ on all objects $W$ in $\mathcal{P}_{l-1}$. Because $Z$ has larger length than $W$, a map $\phi: W \rightarrow Z$ cannot be split epic, so it factors through $\beta$. Hence $\gamma \circ \phi$ is zero, which implies that $\gamma \in \mathcal{I}^{l-1}$.

The inequalities follow from Lemmas 3.1 and Lemma 3.2, with $m=l-1$. The statement about the summand $S$ follows immediately.

Note in particular that for any A-R triangle, the almost zero map $\gamma$ is an example of a non-zero map in the largest possible power of the ideal, for any projective class.

In the case when $\mathbb{T}$ is $\operatorname{StMod}(k G)$ with $G$ being a $p$-group, we know that ghosts and dual ghosts coincide [9]. Hence $\gamma$ non-zero implies that $k \geqslant l$, and so we are in the first case of Proposition 3.6.

In the next section, we develop these ideas further.

\subsection{Auslander-Reiten triangles, irreducible maps and lengths}

In this section, we focus on the category $\operatorname{StMod}(k G)$, and show that there is a close relationship between lengths and irreducible maps.

The category $\operatorname{StMod}(k G)$ satisfies the hypotheses on $\mathbb{T}$ in Theorem 3.4, and its compact objects are precisely the objects of $\operatorname{stmod}(k G)$. For projective-free $M \in \operatorname{stmod}(k G)$, the stable endomorphism ring $\operatorname{End}(M, M)$ being local is equivalent to $M$ being indecomposable. In this case, the AuslanderReiten triangle has the form [2, 4.12.8]

$$
\Omega^{2} M \stackrel{\alpha}{\rightarrow} H(M) \stackrel{\beta}{\rightarrow} M \stackrel{\gamma}{\rightarrow} \Omega M .
$$

The module $H(M)$ is called the heart of $M$, and the triangle shows that it is also in $\operatorname{stmod}(k G)$.

The general theory we have set up in the last two sections applies to an A-R triangle for any projective class $(\mathcal{P}, \mathcal{I})$ on $\operatorname{StMod}(k G)$. As a special case of Proposition 3.6, using that $k=l$ in this case, we get

Corollary 3.7. Let $G$ be a finite group, let $k$ be a field whose characteristic divides the order of $G$, and let $(\mathcal{P}, \mathcal{I})$ be a projective class on $\operatorname{St} \operatorname{Mod}(k G)$. Consider the Auslander-Reiten triangle $\Omega^{2} M \stackrel{\alpha}{\rightarrow} H(M) \stackrel{\beta}{\rightarrow} M \stackrel{\gamma}{\rightarrow} \Omega M$ for some indecomposable non-projective module $M$ in $\operatorname{stmod}(k G)$ with finite length $l$ with respect to $(\mathcal{P}, \mathcal{I})$. Then

$$
\operatorname{len}_{\mathcal{P}}(M)-1 \leqslant \operatorname{len}_{\mathcal{P}}(H(M)) \leqslant \operatorname{len}_{\mathcal{P}}(M)+1
$$

and $\gamma$ is a non-trivial map in $\mathcal{I}^{l-1}$.

As above, we emphasize again that the same map $\gamma: M \rightarrow \Omega M$ provides a map in $\mathcal{I}^{n}$ with $n$ maximal for any projective class $(\mathcal{P}, \mathcal{I})$. Put another way, $\gamma$ is in the intersection of all projective class ideals that contain a non-trivial map from $M$.

Remark 3.8. One might hope that the heart $H(M)$ always has larger generating length than $M$ when $\operatorname{gel}(M)$ is less than the generating number of $k G$, but unfortunately this is not true in general. For example, take $G=C_{5} \times C_{5}$ and $M=k \uparrow_{C_{5}}^{G}$. One can compute that $\operatorname{gel}(M)=\operatorname{gel}(H(M))=5$, while the generating number of $k G$ is at least 6 (Theorem 4.9).

Let $S$ be an indecomposable non-projective summand of $H(M)$. Then, clearly, $\operatorname{len}_{\mathcal{P}}(S) \leqslant$ $\operatorname{len}_{\mathcal{P}}(H(M)) \leqslant \operatorname{len}_{\mathcal{P}}(M)+1$. We will show below that $\operatorname{len}_{\mathcal{P}}(M)-1 \leqslant \operatorname{len}_{\mathcal{P}}(S)$ because of the right minimality of the map $\beta$.

We first need the notion of irreducible map.

Definition 3.9. Let $G$ be a finite group, and let $k$ be a field whose characteristic divides the order of $G$. A map $\lambda: M \rightarrow N$ in $\operatorname{StMod}(k G)$ is said to be irreducible if it is not split monic or split epic, and for any factorization $\lambda=\nu \circ \mu$, either $\mu$ is split monic or $\nu$ is split epic.

Irreducible maps are closely related to Auslander-Reiten triangles:

Proposition 3.10 (Auslander and Reiten [1]). Let $M$ and $N$ be indecomposable non-projective modules in $\operatorname{stmod}(k G)$. Then a map $f: M \rightarrow N$ is irreducible if and only if the following equivalent conditions are satisfied: 
(a) $M$ is a summand of $H(N)$ and $f$ is the composite $M \rightarrow H(N) \stackrel{\beta}{\rightarrow} N$.

(b) $N$ is a summand of $\Omega^{-2} H(M)$ and $f$ is the composite $M \stackrel{\Omega^{-2} \alpha}{\longrightarrow} \Omega^{-2} H(M) \rightarrow N$.

Combining Corollary 3.7 and Proposition 3.10, one can prove

Corollary 3.11. Let $f: M \rightarrow N$ be an irreducible map with $M$ and $N$ non-projective indecomposables in $\operatorname{stmod}(k G)$, and let $(\mathcal{P}, \mathcal{I})$ be a projective class on $\operatorname{StMod}(k G)$. If $M$ and $N$ have finite lengths with respect to $(\mathcal{P}, \mathcal{I})$, then

$$
\operatorname{len}_{\mathcal{P}}(M)-1 \leqslant \operatorname{len}_{\mathcal{P}}(N) \leqslant \operatorname{len}_{\mathcal{P}}(M)+1 .
$$

In particular, for $M$ indecomposable and $S$ any summand of $H(M)$, we have

$$
\operatorname{len}_{\mathcal{P}}(M)-1 \leqslant \operatorname{len}_{\mathcal{P}}(S) \leqslant \operatorname{len}_{\mathcal{P}}(M)+1 .
$$

\subsection{Ghost lengths}

The results of Sections 3.1 to 3.3 apply to the generating length of a module in $\operatorname{StMod}(k G)$, since generating length is the length with respect to the ghost projective class. When $k G$ has periodic cohomology, there is a projective class on Thick $\langle k\rangle$ whose ideal is $\mathcal{G}_{t}$, and ghost length is the length with respect to this projective class. In general, we don't know whether ghost length is a length with respect to a projective class, but we can still prove the analogue of half of Corollary 3.7:

Proposition 3.12. Let $G$ be a finite group, and let $k$ be a field whose characteristic divides the order of $G$. Consider the Auslander-Reiten triangle $\Omega^{2} M \rightarrow H(M) \rightarrow M \rightarrow \Omega M$ for some indecomposable module $M$ in Thick $\langle k\rangle$. Then the following holds:

$$
\operatorname{gl}(M)-1 \leqslant \operatorname{gl}(H(M))
$$

Proof. We mimic the proof of Lemma 3.2. Suppose that $\mathrm{gl}(H(M))=l-1$. We must prove that $\operatorname{gl}(M) \leqslant l$. Since $\operatorname{gl}(M)=\operatorname{gl}\left(\Omega^{2} M\right)$, it suffices to show that any map $\phi: \Omega^{2} M \rightarrow N$ in $\left(\mathcal{G}_{t}\right)^{l}$ is stably trivial, where $\mathcal{G}_{t}$ consists of ghosts between objects in Thick $\langle k\rangle$. Write $\phi$ as $\phi_{2} \phi_{1}$, where $\phi_{1}$ is in $\mathcal{G}_{t}$ and $\phi_{2}$ is in $\left(\mathcal{G}_{t}\right)^{l-1}$. Then, by Proposition 3.6, the composite $\phi_{1} \Omega \gamma$ is stably trivial, so $\phi_{1}$ factors through $H(M)$ :

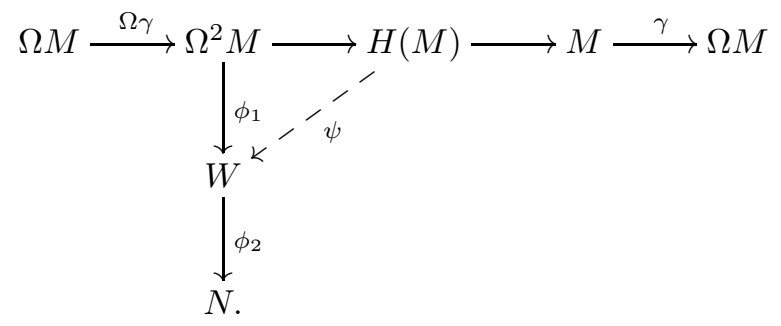

Now since $\operatorname{gl}(H(M))=l-1$, the composite $\phi_{2} \psi$ is stably trivial and so $\phi$ is stably trivial as well.

The analogue of the other half of Corollary 3.7 would say that $\operatorname{gl}(H(M)) \leqslant \operatorname{gl}(M)+1$, and we don't know whether this is true.

Remark 3.13. A related question is whether the generating length and ghost length always agree. We know of no counterexamples. However, Corollary 3.7 implies that the longest composite of ghosts starting from a given module $M$ in Thick $\langle k\rangle$ can always be attained by a map in $\left(\mathcal{G}^{m}\right)_{t}$, the intersection of $\mathcal{G}^{m}$ and Thick $\langle k\rangle$. Thus if $\left(\mathcal{G}_{t}\right)^{m}=\left(\mathcal{G}^{m}\right)_{t}$, then the ghost length and generating length agree. Note that a related statement for the objects of $\mathcal{P}$, i.e., that $\left(\mathcal{P}^{c}\right)_{n}=\left(\mathcal{P}_{n}\right)^{c}$, where the superscript $c$ means to take the intersection with the compact objects, is known to be true $\mathbb{4}, 2.2 .4]$. 


\section{Ghost numbers of $p$-Groups}

In this section we study finite $p$-groups, using the fact that Thick $\langle k\rangle=\operatorname{stmod}(k G)$. We begin in Section 4.1 by recalling several results that we will use. In Section 4.2 we show that the generating length invariant is a stabilized version of the socle length, and give a result that shows that if these are equal for a module $M$, the same is true for $\operatorname{rad}(M)$ and $M / \operatorname{soc}(M)$. Then we give new computations of bounds on ghost numbers for various $p$-groups: abelian $p$-groups in Section 4.3, the quaternion group $Q_{8}$ in Section 4.4, dihedral 2-groups in Section 4.6, and the groups $C_{p^{r}} \times C_{p^{s}}$ in Section 4.7. In several cases we determine the ghost number completely, such as for $D_{4 q}, C_{3} \times C_{3^{s}}$ and $C_{4} \times C_{2^{s}}$. In Section 4.5, we compute the ghost length and generating length of certain modules induced up from a cyclic normal subgroup. This is used in the same section to show that the ghost number and the radical length are within a factor of three of each other for any p-group. It is also used in Section 4.6 in the computation of the ghost number of $k D_{4 q}$ and in Section 4.8 , where we classify group algebras with small ghost number and put constraints on which ghost numbers can occur.

When we write " $p$-group", we always mean "finite $p$-group".

\subsection{Background}

We recall the following theorem, and then explain the terminology and give an idea of the proof.

Theorem 4.1 (Chebolu, Christensen and Mináč [9]). Let $G$ be a p-group, and let $k$ be a field of characteristic $p$. Then the generating length of a $k G$-module $M$ is at most its radical length, and the following inequalities hold:

ghost number of $k G \leqslant$ generating number of $k G<$ nilpotency index of $J(k G) \leqslant|G|$.

In particular, the ghost number of $k G$ is finite in this case.

Let $G$ be any finite group, and let $k$ be a field whose characteristic divides the order of $G$. Let $J=J(k G)$ be the Jacobson radical of $k G$, i.e., the largest nilpotent ideal of $k G$. The nilpotency index of $J(k G)$ is the smallest integer $m$ such that $J^{m}=0$, and for any module $M$, we have a radical series

$$
M=\operatorname{rad}^{0}(M) \supseteq \operatorname{rad}^{1}(M) \supseteq \operatorname{rad}^{2}(M) \supseteq \cdots \supseteq 0,
$$

with $\operatorname{rad}^{n}(M)=J^{n} M$, and a socle series

$$
0=\operatorname{soc}^{0}(M) \subseteq \operatorname{soc}^{1}(M) \subseteq \operatorname{soc}^{2}(M) \subseteq \cdots \subseteq M
$$

with $\operatorname{soc}^{n}(M)$ consisting of the elements of $M$ annihilated by $J^{n}$. The radical length of $M$ is the smallest integer $n$ such that $\operatorname{rad}^{n}(M)=0$. This is equal to the socle length of $M$, the smallest integer $m$ such that $\operatorname{soc}^{m}(M)=M$. The successive quotients in the sequences are direct sums of simple modules.

If $G$ is a $p$-group, then each quotient is a direct sum of $k$ 's, so the generating length of a module $M$ is less than or equal to its radical length. Note that the nilpotency index of $J(k G)$ is exactly the radical length of $k G$, and if $M$ is a projective-free $k G$-module, it always has smaller radical length than $k G$. The theorem then follows.

The following lemma is proved by studying Tate cohomology in degrees 0 and -1 . We write $\operatorname{rad}(M)$ for $\operatorname{rad}^{1}(M)$ and $\operatorname{soc}(M)$ for $\operatorname{soc}^{1}(M)$.

Lemma 4.2 (Chebolu, Christensen and Mináč [9]). Let $G$ be a p-group, and let $k$ be a field of characteristic p. Let $f: M \rightarrow N$ be a map in $\operatorname{Mod}(k G)$ between projective-free modules $M$ and $N$. Then:

(a) $\operatorname{soc}(M) \subseteq \operatorname{ker}(f)$ iff $[k, f]=0$.

(b) $\operatorname{im}(f) \subseteq \operatorname{rad}(N)$ iff $\left[\Omega^{-1} k, f\right]=0$.

In particular, if $f$ represents a ghost in the stable category, then both inclusions hold.

As a corollary, we get 
Corollary 4.3 (Chebolu, Christensen and Mináč [9]). Let $G$ be a p-group, and let $k$ be a field of characteristic $p$. Let $f: M \rightarrow N$ be a map in $\operatorname{Mod}(k G)$ between projective-free modules $M$ and $N$. If $f$ is an $l$-fold ghost, then:

(a) $\operatorname{soc}^{l}(M) \subseteq \operatorname{ker}(f)$.

(b) $\operatorname{im}(f) \subseteq \operatorname{rad}^{l}(N)$.

The next lemma provides ghosts with a particular form.

Lemma 4.4 (Benson, Chebolu, Christensen and Mináč [3]). Let $G$ be a p-group, and let $k$ be a field of characteristic $p$. Let $x \in G$ be a central element. Then left multiplication by $x-1$ on a $k G$-module $M$ is a ghost.

Note that in general there are ghosts not of this form. Nevertheless these ghosts work well for abelian groups in providing lower bounds for ghost numbers (see Section 1.3). It is not hard to check that if $G$ is a cyclic $p$-group with generator $g$, then $g-1$ is a universal ghost.

\subsection{Generating and socle lengths}

We now show that the generating length is a stabilized version of the socle length. In this section we allow our projective classes to be unstable, that is, we don't assume that the projectives are closed under suspension and desuspension.

Let $G$ be a $p$-group, let $k$ be a field of characteristic $p$, and let $M$ be a $k G$-module. Note that $\operatorname{soc}(M)$ contains exactly the image of maps from $k$. So, when we build up $M$ in a socle sequence in Theorem 4.1, we are only using maps from $k$, not all suspensions of $k$. This suggests that we consider the unstable projective class generated by $k$ in $\operatorname{StMod}(k G)$. We will show that the length with respect to this projective class is exactly the socle length for projective-free modules in $\operatorname{stmod}(k G)$.

Note that the regular representation $k G$ is the only indecomposable projective $k G$-module, and $\operatorname{soc}(k G) \cong k$ is its unique minimal left submodule. Thus any map $k G \rightarrow M$ in $\operatorname{Mod}(k G)$ with $M$ projective-free has $\operatorname{soc}(k G)$ in its kernel, since the map cannot be injective. It follows that a map $\oplus k \rightarrow M$ in $\operatorname{Mod}(k G)$ with $M$ projective-free is stably trivial if and only if it is the zero map. For finitely generated modules, a similar argument shows that the same is true for a map $M \rightarrow \oplus k$ in $\bmod (k G)$ with $M$ projective-free.

Proposition 4.5. Let $G$ be a p-group, and let $k$ be a field of characteristic $p$. Let $(\mathcal{P}, \mathcal{I})$ be the unstable projective class in $\operatorname{StMod}(k G)$ generated by $k$. Then a map $f: M \rightarrow N$ between projectivefree objects $M$ and $N$ is in $\mathcal{I}$ if and only if it is represented by a map $f$ such that $\operatorname{soc}(M) \subseteq \operatorname{ker}(f)$. Hence, if $M$ is finitely-generated and projective-free, the length of $M$ with respect to $(\mathcal{P}, \mathcal{I})$ is exactly its socle length.

Proof. That $f \in \mathcal{I}$ is equivalent to $\operatorname{soc}(M) \subseteq \operatorname{ker}(f)$ is Lemma 1.2 (a).

Now let $M$ be projective-free. Then $M \rightarrow M / \operatorname{soc}(M)$ is a universal map in $\mathcal{I}$. It follows that $M \rightarrow M / \operatorname{soc}^{k}(M)$ is universal in $\mathcal{I}^{k}$. If $M$ has socle length $n$, then $M \in \mathcal{P}^{n}$ and $M \rightarrow M / \operatorname{soc}^{n-1}(M)$ is non-zero. If further $M$ is finitely-generated, then the universal map $M \rightarrow M / \operatorname{soc}^{n-1}(M) \cong \oplus k$ is stably non-trivial, by the remarks preceding this proposition. Thus $M$ has length $n$ with respect to $(\mathcal{P}, \mathcal{I})$.

Note that the stable projective class generated by $k$ in $\operatorname{StMod}(k G)$ is exactly the ghost projective class. Thus the generating length is indeed the socle length stabilized and is generally less than or equal to the socle length. We have also recovered Theorem 4.1 from this observation. In Section 4.5, we are going to prove that the generating number of $k G$ is within a factor of 3 of the socle length of $k G$.

Here we show that if the generating length of a module $M \in \operatorname{StMod}(k G)$ happens to equal its socle length (see, for example, Proposition 4.10 and Theorem 4.15), then the same holds for $\operatorname{rad}(M)$ and $M / \operatorname{soc}(M)$, a result that we will use in Section 4.6 when studying dihedral groups. 
Proposition 4.6. Let $k$ be a field of characteristic $p$, and let $G$ be a p-group. Assume that $M \in$ $\operatorname{StMod}(k G)$ has generating length equal to its radical length. Then $\operatorname{gel}(M / \operatorname{soc}(M))=\operatorname{gel}(M)-1$, and similarly $\operatorname{gel}(\operatorname{rad}(M))=\operatorname{gel}(M)-1$.

Proof. Since the generating length of $M$ is strictly less than the nilpotency index of $J(k G), M$ is projective-free. The proposition is then a special case of the following more general lemma.

Lemma 4.7. Let $\mathbb{T}$ be a triangulated category, and let $(\mathcal{P}, \mathcal{I})$ and $\left(\mathcal{P}^{\prime}, \mathcal{I}^{\prime}\right)$ be (possibly unstable) projective classes on $\mathbb{T}$ such that $\mathcal{P}^{\prime} \subseteq \mathcal{P}$. Suppose that $M \in \mathbb{T}$ has $\operatorname{len}_{\mathcal{P}^{\prime}}(M)=\operatorname{len}_{\mathcal{P}}(M)=m$ and that there exist $L \in \mathcal{P}_{m-n}^{\prime}$ and $N \in \mathcal{P}_{n}^{\prime}$ with a triangle

$$
L \rightarrow M \rightarrow N
$$

Then

$$
\operatorname{len}_{\mathcal{P}^{\prime}}(L)=\operatorname{len}_{\mathcal{P}}(L)=m-n \text {, and } \operatorname{len}_{\mathcal{P}^{\prime}}(N)=\operatorname{len}_{\mathcal{P}}(N)=n .
$$

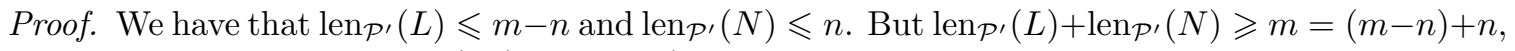
so the equalities follow for $\left(\mathcal{P}^{\prime}, \mathcal{I}^{\prime}\right)$. Since $\mathcal{P}^{\prime} \subseteq \mathcal{P}$, the same results hold for $(\mathcal{P}, \mathcal{I})$ too.

Intuitively, this easy fact says that when $\operatorname{len}_{\mathcal{P}^{\prime}}(M)=\operatorname{len}_{\mathcal{P}}(M)$, the related object $L$ can be built from $\mathcal{P}^{\prime}$ as efficiently as it can be built from $\mathcal{P}$. It applies to generating lengths and socle lengths.

We now provide examples of computations of ghost numbers of certain groups, improving on results in [9].

\subsection{Ghost numbers of abelian $p$-groups}

We first prove a general proposition. It generalizes [3, Lemma 2.3] and [9, Prop. 5.10].

Proposition 4.8. Let $k$ be a field of characteristic $p$, and let $H$ be a non-trivial subgroup of a p-group $G$. Assume that there exists a central element $x$ in $G$. Let $l$ be the smallest positive integer such that $x^{l} \in H$. Suppose that $M \in \operatorname{StMod}(k H)$ has generating length $m \geqslant 1$. Then $\operatorname{gel}\left(M \uparrow^{G}\right) \geqslant$ $\operatorname{gel}(M)+(l-1)$, and

$$
\text { generating number of } k G \geqslant \text { generating number of } k H+(l-1) \text {. }
$$

Suppose that $M \in \operatorname{stmod}(k H)$ has ghost length $n \geqslant 1$. Then $\operatorname{gl}(M \uparrow G) \geqslant \operatorname{gl}(M)+(l-1)$, and

$$
\text { ghost number of } k G \geqslant \text { ghost number of } k H+(l-1) \text {. }
$$

Proof. For brevity, we write $\downarrow$ for $\downarrow_{H}^{G}$ and $\uparrow$ for $\uparrow_{H}^{G}$. Let $f: M \rightarrow N$ be a non-trivial $(m-1)$-fold ghost in $\operatorname{StMod}(k H)$. We will show that $(x-1)^{l-1} \circ f \uparrow$ is stably non-trivial. Since ghosts induce up to ghosts and $x-1$ is a ghost, it follows that there exists a non-trivial composite of $(m-1)+(l-1)$ ghosts in $\operatorname{StMod}(k G)$.

Consider the map $M \stackrel{i}{\rightarrow} M \uparrow \downarrow \stackrel{f \uparrow \downarrow}{\longrightarrow} N \uparrow \downarrow \stackrel{(x-1)^{l-1} \downarrow}{\longrightarrow} N \uparrow \downarrow \stackrel{r}{\rightarrow} N$, where $i$ and $r$ are the natural maps. To be more explicit, $M \uparrow_{H}^{G}=k G \otimes_{H} M, i(\alpha)=1 \otimes \alpha$ and $r(g \otimes \alpha)=g \alpha$ if $g \in H$ and is zero otherwise. By naturality of the inclusion, the composite equals $M \stackrel{f}{\rightarrow} N \stackrel{i}{\rightarrow} N \uparrow \downarrow \stackrel{(x-1)^{l-1} \downarrow}{\longrightarrow} N \uparrow \downarrow \stackrel{r}{\rightarrow} N$. Since $x^{i} \notin H$ for $i \leqslant l-1$, the map $N \stackrel{i}{\rightarrow} N \uparrow \downarrow \stackrel{(x-1)^{l-1} \downarrow}{\longrightarrow} N \uparrow \downarrow \stackrel{r}{\rightarrow} N$ is simply multiplication by $(-1)^{l-1}$, an isomorphism. Since $N$ is stably non-zero, it follows that $(x-1)^{l-1} \downarrow \circ f \uparrow \downarrow$ and therefore $(x-1)^{l-1} \circ f \uparrow$ are stably non-trivial.

The result on ghost length and ghost number can be proved similarly by replacing $\operatorname{StMod}(k G)$ with $\operatorname{stmod}(k G)$.

We can apply this proposition to abelian groups. 
Theorem 4.9. Let $k$ be a field of characteristic $p$, and let $A=C_{p^{r}} \times C_{p^{r_{1}}} \times \cdots \times C_{p^{r_{l}}}$ be an abelian p-group. Then

$$
m-p^{r}+\left\lceil\frac{p^{r}-1}{2}\right\rceil \leqslant \text { ghost number of } k A \leqslant \text { generating number of } k A \leqslant m-1,
$$

where $m$ is the nilpotency index of $J(k A)$, and $p^{r}$ is the order of the smallest cyclic summand.

When the prime $p$ is greater than 2, the result here improves on that in [9], where the lower bound for the ghost number of $k A$ is given by $m-p^{r}+p^{r-1}=m-p^{r}+\left\lceil\left(p^{r}-1\right) / p\right\rceil$.

Note that since

$$
m=1+\left(p^{r}-1\right)+\left(p^{r_{1}}-1\right)+\cdots+\left(p^{r_{l}}-1\right),
$$

our lower bound can also be written as

$$
\left\lceil\frac{p^{r}-1}{2}\right\rceil+\left(p^{r_{1}}-1\right)+\cdots+\left(p^{r_{l}}-1\right) .
$$

Also note that when $A$ is cyclic, we have $m=p^{r}$, and the lower bound $d=\left\lceil\frac{p^{r}-1}{2}\right\rceil$ here is exactly the ghost number of $A$ [9, Thm. 5.4].

Proof. Let $g$ be a generator of $C_{p^{r}}$, and let $g_{i}$ be a generator of $C_{p^{r_{i}}}, i=1,2, \ldots, l$. Write $d=\left\lceil\frac{p^{r}-1}{2}\right\rceil$. By the proof of [9, Prop. 5.3], $k C_{p^{r}}$ has ghost number $d$. We can now apply Proposition 4.8 by successively including the summands $C_{p^{r_{i}}}$ to obtain

$$
\text { ghost number of } k A \geqslant d+\left(p^{r_{1}}-1\right)+\cdots+\left(p^{r_{l}}-1\right) \text {. }
$$

The other inequalities are from Theorem 4.1.

Proposition 4.8 allows us to make this explicit. Let $M=N \uparrow_{C_{p^{r}}}^{A}$, with $N=k C_{p^{r}} /(g-1)^{d}$. Note that $(g-1)^{d-1}$ is a stably non-trivial $(d-1)$-fold ghost on $N$ in $\operatorname{stmod}\left(k C_{p^{r}}\right)$ and, since $A$ is abelian, the self map $(g-1) \uparrow_{C_{p^{r}}}^{A}$ on $M$ is simply left multiplication by $g-1$. Hence we have a particular form for the non-trivial $\left(m-p^{r}+d-1\right)$-fold ghost on $M$ :

$$
\theta=(g-1)^{d-1}\left(g_{1}-1\right)^{p^{r_{1}}-1} \cdots\left(g_{l}-1\right)^{p^{r_{l}}-1} .
$$

More generally, we have the following result.

Proposition 4.10. Let $k$ be a field of characteristic p, let $A=C_{p^{r_{1}}} \times C_{p^{r_{2}}} \times \cdots \times C_{p^{r_{l}}}$ be an abelian p-group, and let $M_{i}$ be an indecomposable $C_{p^{r_{i}}}$-module of dimension $n_{i}$ for each $i$. Then the $A$-module $M=M_{1} \otimes \cdots \otimes M_{l}$ has radical length $1+\left(n_{1}-1\right)+\cdots+\left(n_{l}-1\right)$. If $n_{i} \leqslant \frac{p^{r_{i}}}{2}$ for some $i$, then the generating length of $M$ equals its radical length.

Before proving the proposition, we state the following lemma.

Lemma 4.11 ([14, Theorem 1.2]). Let $G$ be a p-group, and let $k$ be a field of characteristic $p$. Then the elements $h-1$ with $h \neq 1$ form a basis for $\operatorname{rad}(k G)$. It follows that the products $\left(h_{1}-1\right) \cdots\left(h_{n}-1\right)$ with $h_{i} \neq 1$ span $\operatorname{rad}^{n}(k G)$.

Note that it suffices to consider generators of the group $G$ when we generate $\operatorname{rad}^{n} k G$ as a submodule. We can now compute the radical length of the module $M$ and prove the proposition.

Proof of Proposition. Let $g_{i}$ be a generator of $C_{p^{r_{i}}}$. Then the various $g_{i}-1$ with $1 \leqslant i \leqslant l$ generate $\operatorname{rad}(k G)$. We regard $M_{i}$ as the quotient $k C_{p^{r_{i}}} /\left(g_{i}-1\right)^{n_{i}}$, so the elements $\left(g_{i}-1\right)^{j}$ with $0 \leqslant j \leqslant n_{i}-1$ form a basis of $M_{i}$. Now let $m=\left(n_{1}-1\right)+\cdots+\left(n_{l}-1\right)$. Since any $(m+1)$-fold product of the elements $g_{i}-1$ has to be zero in $M, \operatorname{rad}^{m+1}(M)=0$. On the other hand, the element $\left(g_{1}-1\right)^{n_{1}-1} \otimes \cdots \otimes\left(g_{l}-1\right)^{n_{l}-1} \in M$ is non-zero and spans $\operatorname{rad}^{m}(M)$. It follows that the radical length of $M$ is $m+1$. 
To prove the last statement, without loss of generality we can assume that $n_{1} \leqslant \frac{p^{r_{1}}}{2}$. We then consider the restriction of $M$ to $H=C_{p^{r_{1}}}$. Note that we have a vector space isomorphism

$$
M \downarrow_{H} \cong \bigoplus_{i_{2}=0}^{n_{2}-1} \cdots \bigoplus_{i_{l}=0}^{n_{l}-1} M_{1} .
$$

Since $G$ acts componentwise, this is actually an isomorphism of $k H$-modules, and we have $k H$-maps $i: M_{1} \rightarrow M \downarrow_{H}$ sending $\alpha$ to $\alpha \otimes 1 \otimes \cdots \otimes 1$ and $r: M \downarrow_{H} \rightarrow M_{1}$ sending $\alpha \otimes\left(g_{2}-1\right)^{i_{2}} \otimes \cdots \otimes\left(g_{l}-1\right)^{i_{l}}$ to $(-1)^{i_{2}+\cdots+i_{l}} \alpha$ for $0 \leqslant i_{k} \leqslant n_{k}-1$.

We can form the $m$-fold ghost $f=\left(g_{1}-1\right)^{n_{1}-1} \cdots\left(g_{l}-1\right)^{n_{l}-1}$ on $M$. And one can check that $r \circ f \downarrow_{H} \circ i$ is $\pm\left(g_{1}-1\right)^{n_{1}-1}$ on $M_{1}$, which is stably non-trivial. Hence $f$ is stably non-trivial and the ghost length of $M$ is at least $m+1$. Since this is also the radical length of $M$, we have $\operatorname{gl}(M)=\operatorname{gel}(M)=m+1$.

Remark 4.12. We don't know which of the lower bound and upper bound better approximates the ghost number in general, but we suspect that the lower bound is better. We show in Section 4.7 that the upper bound can be refined by 1 for rank 2 abelian $p$-groups $C_{p^{r}} \times C_{p^{s}}$, with $p^{r}, p^{s} \geqslant 3$. In particular, the lower bound we have here is the exact ghost number for the group $C_{3} \times C_{3}$.

\subsection{Ghost number of the quaternion group $Q_{8}$}

In this section, we study the quaternion group $Q_{8}=\left\langle\epsilon, i, j \mid \epsilon^{2}=1, i^{2}=j^{2}=(i j)^{2}=\epsilon\right\rangle$ over a field $k$ of characteristic 2. It has been shown in [9] that the ghost number of $k Q_{8}$ is 2,3 , or 4 .

Proposition 4.13. Let $k$ be a field of characteristic 2. Then there exists a stably non-trivial double ghost in $\operatorname{stmod}\left(k Q_{8}\right)$. Hence

$$
3 \leqslant \text { ghost number of } k Q_{8} \leqslant \text { generating number of } k Q_{8} \leqslant 4 .
$$

Proof. We have a quotient map from $Q_{8}$ to the Klein four group $V$ that identifies $\epsilon$ with 1 . We also write $i$ and $j$ for the generators of $V$. The rank one free $k V$-module can be viewed as a $k Q_{8}$-module, and we write $k V$ for it. It has radical length 3 , and we will show that it admits a stably non-trivial double ghost, hence $\operatorname{gl}(k V)=\operatorname{gel}(k V)=3$.

Right multiplication $R_{i+1}$ on $k V$ by $i+1$ is a left $k Q_{8}$-map, and we claim that it is a ghost. To see this, consider the short exact sequence

$$
0 \rightarrow k V \stackrel{\iota}{\rightarrow} k Q_{8} \rightarrow k V \rightarrow 0
$$

of left $k Q_{8}$-modules, where the kernel $k V$ is generated by $\epsilon+1$ in $k Q_{8}$. It follows from this sequence that $\Omega k V=k V$ and that $\Omega R_{i+1}=R_{i+1}$.

Thus to show that $R_{i+1}$ is a ghost, we just need to check that it is stably trivial on maps from $k$. Multiplication by $i+1$ kills the socle of $k V$, which is generated by $1+i+j+i j$, so this follows from Lemma 4.2(a).

Next we show that there is a non-trivial double ghost. For any map $f: k Q_{8} \rightarrow k V$, the composite $f \iota$ is zero, since $\epsilon+1$ acts trivially on $k V$. Thus a $k Q_{8}$-map $k V \rightarrow k V$ is stably trivial if and only if it is zero. As a result, multiplication by $(i+1)(j+1)$ on $k V$ is stably non-trivial, and we get the desired double ghost.

It follows that the ghost number of $k Q_{8}$ is at least 3. The nilpotency index of $J\left(k Q_{8}\right)$ is 5 , so the generating number of $k Q_{8}$ is at most 4 .

Remark 4.14. The map $R_{(i+1)(j+1)}=R_{1+i+j+i j}: k V \rightarrow k V$ constructed in the proof is in fact the almost zero map with domain $k V$ in $\operatorname{stmod}\left(k Q_{8}\right)$. To see this, we consider the inclusion $\operatorname{rad}(k V) \rightarrow$ $k V$. Since this map is not split-epi, its composition with the almost zero map $\gamma: k V \rightarrow k V$ factors through a projective module $P$. But $P$ is also injective, thus we can change $\gamma$ by a map factoring through $P$ to ensure that $\operatorname{rad}(k V) \subseteq \operatorname{ker}(\gamma)$. Since $k V / \operatorname{rad}(k V) \cong \operatorname{soc}(k V) \cong k$ and $\operatorname{soc}(k V)$ is generated by the element $1+i+j+i j$, it must be that $R_{1+i+j+i j}$ is the almost zero map (up to a scalar factor). This gives another proof that this map is stably non-trivial. 
In the next section, we generalize the technique used here.

\section{5. $p$-groups with cyclic normal subgroups}

In Section 4.3, we produced ghosts using left multiplication by $x-1$ for abelian groups. More generally, in Lemma 4.4, we saw that left multiplication by $x-1$ for $x$ a central element produces a ghost. For a non-central element, in order to produce a left module map, one must consider right multiplication, when this makes sense, and indeed we used this technique in Section 4.4 to produce ghosts for $Q_{8}$. However, it is not always true that right multiplication by $x-1$ produces ghosts. Generalizing the known examples, we show that if $M$ is induced up from a cyclic normal subgroup, then right multiplication by $x-1$ on $M$ is well-defined and is a ghost.

Theorem 4.15. Let $C_{p^{r}}$ be a cyclic normal subgroup of a p-group $G$, and let $k$ be a field of characteristic $p$. Let $M_{n}$ be an indecomposable $k C_{p^{r}}$-module of dimension $n$, and write $M=M_{n} \uparrow^{G}$. Then, for each $x \in G$, one can define the right multiplication map $R_{x-1}$ on $M$ and it is a ghost. Moreover, if $n \leqslant\left\lceil\frac{p^{r}-1}{2}\right\rceil$, then $\operatorname{gl}(M)=\operatorname{gel}(M)=$ radlen $M$.

Note that for $n=1$, we have $M \cong k H \downarrow_{G}$, where $H=G / C_{p^{r}}$ and the restriction is taken along the quotient map. Thus the ghosts in the previous section are examples of this construction.

Proof. Let $g$ be a generator of $C_{p^{r}}$. We can identify $M_{n}$ with the left submodule of $k C_{p^{r}}$ generated by $(g-1)^{p^{r}-n}$, and so we have a short exact sequence of $k C_{p^{r}}$-modules:

$$
0 \rightarrow M_{n} \rightarrow k C_{p^{r}} \rightarrow M_{p^{r}-n} \rightarrow 0,
$$

where $M_{p^{r}-n}$ is an indecomposable $k C_{p^{r}}$-module of dimension $p^{r}-n$. Inducing up, we get

$$
0 \rightarrow M_{n} \uparrow^{G} \stackrel{i}{\longrightarrow} k G \stackrel{p}{\longrightarrow} M_{p^{r}-n} \uparrow^{G} \rightarrow 0 .
$$

The inclusion $i$ identifies $M=M_{n} \uparrow^{G}$ with the left submodule of $k G$ generated by $(g-1)^{p^{r}-n}$. Since $C_{p^{r}} \leqslant G$ is normal, this submodule is actually a sub-bimodule. Thus the right multiplication map $R_{x-1}: M \rightarrow M$ is well-defined and is a left $k G$-module map, for each $x \in G$. We must show that it is a ghost.

Since (4.1) is in fact a short exact sequence of bimodules, $R_{x-1}$ is two-periodic as a left $k G$-map, so it suffices to check that $R_{x-1}$ is left stably-trivial on maps from $k$ and $\Omega^{-1} k$. By Lemma 1.2, this is equivalent to $\operatorname{soc}_{L}(M) \subseteq \operatorname{ker}\left(R_{x-1}\right)$ and $\operatorname{im}\left(R_{x-1}\right) \subseteq \operatorname{rad}_{L}(M)$, where we use subscripts to indicate left and right socles and radicals. Clearly, $\operatorname{soc}_{R}(M) \subseteq \operatorname{ker}\left(R_{x-1}\right)$ and $\operatorname{im}\left(R_{x-1}\right) \subseteq \operatorname{rad}_{R}(M)$.

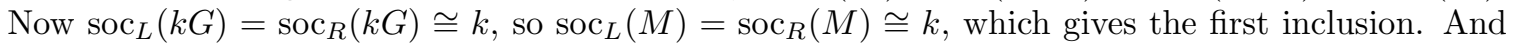
one can also show that $\operatorname{rad}_{L}(M)=\operatorname{rad}_{R}(M)$, which gives the second inclusion.

To prove the last claim, let $n \leqslant\left\lceil\frac{p^{r}-1}{2}\right\rceil$ and assume that rad len $M=l$. We want to construct an $(l-1)$-fold ghost. Note that $\operatorname{soc}_{L}(M)=\operatorname{soc}_{R}(M)=\operatorname{rad}_{R}^{l-1}(M)=M\left(g_{1}-1\right) \cdots\left(g_{l-1}-1\right)$ for some $g_{1}, \ldots, g_{l-1}$ in $G$, so the $(l-1)$-fold ghost $f:=R_{g_{l-1}-1} \circ \cdots \circ R_{g_{1}-1}$ takes $M$ onto its socle. For any map $h: k G \rightarrow M$, the composite $h i$ is zero, since the image of $i$ is generated by $(g-1)^{p^{r}-n}$ which acts trivially on $M$ since $n \leqslant p^{r}-n$. Thus a map $M \rightarrow M$ is stably trivial if and only if it is zero, and so our $(l-1)$-fold ghost $f$ is stably non-trivial. Thus $l \leqslant \operatorname{gl}(M) \leqslant \operatorname{gel}(M) \leqslant \operatorname{radlen}(M)=l$, and we are done.

Remark 4.16. As in Remark 4.14, we can also see that $f$ is non-trivial using the theory of AuslanderReiten triangles. There is a canonical inclusion $j$ of $M$ into $M_{p^{r}-n} \uparrow^{G}=\Omega M$ induced from the $k C_{p^{r}}$-map $M_{n} \rightarrow M_{p^{r}-n}$, and one can show that the composite $j f$ is exactly the almost zero map out of $M$.

Note that any $p$-group $G$ has a non-trivial center, hence a cyclic normal subgroup $C_{p}$. Applying the theorem to the short exact sequence of groups $C_{p} \rightarrow G \rightarrow H$, we get 
Corollary 4.17. Let $G$ be a p-group, and let $k$ be a field of characteristic $p$. Then

$$
\frac{1}{2} \text { radlen } k G \leqslant \text { ghostnum } k G \leqslant \text { gen num } k G<\operatorname{radlen~} k G,
$$

when $p$ is even, and

$$
\frac{1}{3} \text { radlen } k G \leqslant \text { ghostnum } k G \leqslant \text { gen num } k G<\operatorname{radlen~} k G,
$$

when $p$ is odd.

Proof. Choose a cyclic normal subgroup $C_{p}$ of $G$, and let $M=M_{n} \uparrow^{G}$, where $M_{n}$ is an indecomposable $k C_{p}$-module of dimension $n=\left\lceil\frac{p-1}{2}\right\rceil$. Since $\operatorname{rad}$ len $M=\operatorname{gl}(M) \leqslant \operatorname{ghost}$ num $k G$, we only need to show that $2(\operatorname{radlen} M) \geqslant \operatorname{radlen} k G$ for $p$ even and $3(\operatorname{radlen} M) \geqslant \operatorname{rad}$ len $k G$ for $p$ odd. By (4.1), we know that $\operatorname{rad}$ len $M+\operatorname{rad}$ len $M_{p-n} \uparrow^{G} \geqslant \operatorname{rad}$ len $k G$.

For $p$ even, $p-n=n$, and so the result follows.

For $p$ odd, $p-n=n+1$. We will show that $2(\operatorname{radlen} M) \geqslant \operatorname{rad}$ len $M_{n+1} \uparrow^{G}$, and the corollary will follow. There is a short exact sequence

$$
0 \rightarrow M \rightarrow M_{n+1} \uparrow^{G} \rightarrow M_{1} \uparrow^{G} \rightarrow 0,
$$

induced up from $C_{p}$-maps, and one sees that $M_{1} \uparrow{ }^{G}$ is a submodule of $M$ again by inducing up the $C_{p}$-map $k \rightarrow M_{n}$. It follows that $2(\operatorname{rad}$ len $M) \geqslant \operatorname{rad}$ len $M+\operatorname{rad}$ len $M_{1} \uparrow^{G} \geqslant \operatorname{rad} \operatorname{len} M_{n+1} \uparrow^{G}$.

We expect that for odd primes, the lower bound can be improved to an expression that is generically close to $(\operatorname{rad} \operatorname{len} k G) / 2$.

\subsection{Ghost numbers of dihedral 2-groups}

Our next goal is to study the dihedral 2-groups. We will show that the ghost number and generating number of $k D_{4 q}$ are both $q+1$. Here we write $D_{4 q}$ for the dihedral 2-group of order $4 q$, with $q$ a power of 2 :

$$
D_{4 q}=\left\langle x, y \mid x^{2}=y^{2}=1,(x y)^{q}=(y x)^{q}\right\rangle .
$$

It has a normal cyclic subgroup $C_{2 q}$, generated by $g=x y$.

Since $k C_{2 q}$ has ghost number $q$, which is realized by the ghost length of $M=k C_{2 q} /(g-1)^{q}$, Prop. 5.3], the ghost length of $N=M \uparrow_{C_{2 q}}^{D_{4 q}}$ is at least $q$ in $\operatorname{stmod}\left(k D_{4 q}\right)$. By Theorem 4.15, we actually have $\operatorname{gl}(N)=\operatorname{gel}(N)=\operatorname{rad}$ len $N$. Note that $(x y)^{q} \in D_{4 q}$ is central of order 2 and that $M \cong k \uparrow_{C_{2}}^{C_{2 q}}$, hence $N=M \uparrow_{C_{2 q}}^{D_{4 q}} \cong k \uparrow_{C_{2}}^{D_{4 q}} \cong k D_{2 q} \downarrow_{D_{4 q}}^{D_{2 q}}$, where the restriction is along the quotient map in the short exact sequence $C_{2} \rightarrow D_{4 q} \rightarrow D_{2 q}$. It is not hard to see that the radical length of $k D_{2 q}$ is $q+1$ (see Remark 4.20) and that its $q$-th radical is generated by $((y-1)(x-1))^{\frac{q}{2}}=((x-1)(y-1))^{\frac{q}{2}}$ (which makes sense for $q=1$ since we have identified $x=y$ in that case). Thus we have proved the following consequence of Theorem 4.15 :

Corollary 4.18. Let $k$ be a field of characteristic 2 . Then the ghost number of $k D_{4 q}$ is at least $q+1$. In fact, $\operatorname{gl}(N)=\operatorname{gel}(N)=q+1$, where $N=k \hat{\kappa}_{C_{2}}^{D_{4 q}}$.

The proof of Theorem 4.15 shows that an explicit $q$-fold ghost $N \rightarrow N$ is given by the right multiplication map $R_{((x-1)(y-1))^{\frac{q}{2}}}$.

To get upper bounds for the generating numbers of dihedral 2 -groups, we need classification theorems [2].

Let $\Lambda=k\langle X, Y\rangle /\left(X^{2}, Y^{2}\right)$ be the quotient of the free algebra on two non-commuting variables. In $k D_{4 q}$, writing $X=x-1$ and $Y=y-1$, one can show that $(X Y)^{r}-(Y X)^{r}=(x y)^{r}-(y x)^{r}$ for $r$ a power of 2 , and so $k D_{4 q} \cong \Lambda /\left((X Y)^{q}-(Y X)^{q}\right)$ [2, Lemma 4.11.1].

In the isomorphism $k D_{4 q} \cong \Lambda /\left((X Y)^{q}-(Y X)^{q}\right)$, we have implicitly assumed that the characteristic of $k$ is 2 . However, for the classification we describe below, $k$ can have any characteristic, and we apply it in this generality in the next section. 
$\Lambda$-modules are classifiable. Let $\mathcal{W}$ be the set of words in the direct letters $a$ and $b$ and the inverse letters $a^{-1}$ and $b^{-1}$, such that $a$ and $a^{-1}$ are always followed by $b$ or $b^{-1}$ and vice versa, together with the "zero length word" 1 .

Given $C=l_{1} \cdots l_{n} \in \mathcal{W}$, where each $l_{i}$ is a direct or inverse letter, let $M(C)$ be the vector space over $k$ with basis $z_{0}, \ldots, z_{n}$ on which $\Lambda$ acts according to the schema

$$
k z_{0} \stackrel{l_{1}}{\longleftarrow} k z_{1} \stackrel{l_{2}}{\longleftarrow} k z_{2} \cdots k z_{n-1} \stackrel{l_{n}}{\longleftarrow} k z_{n}
$$

with $X$ acting via $a$ and $Y$ acting via $b$. For example, if $C=a b^{-1} a^{-1}$, then the schema is

$$
k z_{0} \stackrel{a}{\leftarrow} k z_{1} \stackrel{b}{\rightarrow} k z_{2} \stackrel{a}{\rightarrow} k z_{3}
$$

and the module $M\left(a b^{-1} a^{-1}\right)$ is given by

$$
X \mapsto\left[\begin{array}{cccc}
0 & 0 & 0 & 0 \\
1 & 0 & 0 & 0 \\
0 & 0 & 0 & 1 \\
0 & 0 & 0 & 0
\end{array}\right] \quad \text { and } \quad Y \mapsto\left[\begin{array}{cccc}
0 & 0 & 0 & 0 \\
0 & 0 & 1 & 0 \\
0 & 0 & 0 & 0 \\
0 & 0 & 0 & 0
\end{array}\right]
$$

with the matrices acting on row vectors on the right. Such a module is called a module of the first kind. Clearly, $M(C) \cong M\left(C^{-1}\right)$, where $C^{-1}$ reverses the order of the letters in $C$ and inverts each letter.

Let $C=l_{1} \cdots l_{n}$ be a word in $\mathcal{W}$ of even non-zero length that is not a power of a smaller word, and let $V$ be a vector space with an indecomposable automorphism $\phi$ on it. An automorphism is indecomposable if its rational canonical form has only one block, and the block corresponds to a power of an irreducible polynomial over $k$. Let $M(C, \phi)$ be the vector space $\oplus_{i=0}^{n-1} V_{i}$, with $V_{i} \cong V$, and let $\Lambda$ act on $M(C, \phi)$ via the schema

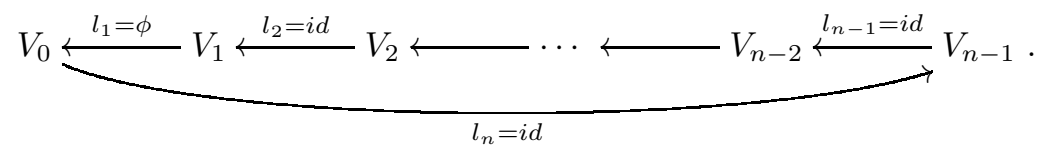

Such a module is called a module of the second kind. Clearly, $M(C, \phi) \cong M\left(C^{-1}, \phi^{-1}\right)$. And if $C^{\prime}$ differs from $C$ by a cyclic permutation, say $l_{1} \cdots l_{n} \mapsto l_{n} l_{1} \cdots l_{n-1}$, then $M(C, \phi) \cong M\left(C^{\prime}, \phi\right)$. Moreover, if $V^{\prime}$ is another vector space with an indecomposable automorphism $\phi^{\prime}$, and $V \cong V^{\prime}$ via an isomorphism that commutes with $\phi$ and $\phi^{\prime}$, then $M(C, \phi) \cong M\left(C^{\prime}, \phi^{\prime}\right)$.

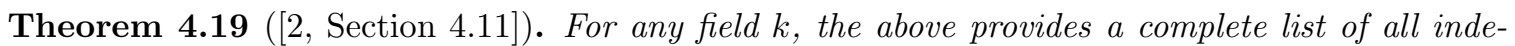
composable $\Lambda$-modules, up to isomorphism. One of these modules has $(X Y)^{q}-(Y X)^{q}$ in its kernel if and only if one of the following holds:

(a) The module is of the first kind and the corresponding word does not contain $(a b)^{q},(b a)^{q}$, or their inverses.

(b) The module is of the second kind and no power of the corresponding word contains $(a b)^{q},(b a)^{q}$, or their inverses.

(c) The module is $M\left((a b)^{q}(b a)^{-q}, i d\right)$. It is a module of the second kind and is the projective indecomposable module for the algebra $\Lambda /\left((X Y)^{q}-(Y X)^{q}\right)$.

Thus, when $k$ has characteristic 2 , a complete list of indecomposable $k D_{4 q}$-modules, up to isomorphism, consists of the $\Lambda$-modules satisfying one of these three conditions.

Remark 4.20. The fact that $k D_{4 q} \cong \Lambda /\left((X Y)^{q}-(Y X)^{q}\right)$ yields that $k D_{4 q}=M\left((a b)^{q}(b a)^{-q}, i d\right)$. It is not hard to see from the schema of $M\left((a b)^{q}(b a)^{-q}, i d\right)$ that it has radical length $2 q+1$. Here is an illustration for $q=2$ : 


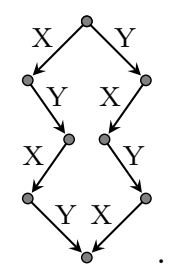

The module $N=k \uparrow_{C_{2}}^{D_{4 q}}=k D_{4 q} \otimes_{k C_{2}} k$ is the quotient of $k D_{4 q}$ where we identify $(x y)^{q}$ with 1 , in other words, $(x y)^{\frac{q}{2}}=(y x)^{\frac{q}{2}}$, for $q>1$. This is equivalent to $(X Y)^{\frac{q}{2}}=(Y X)^{\frac{q}{2}}$. Hence $N=M\left((a b)^{\frac{q}{2}}(b a)^{-\frac{q}{2}}, i d\right)$ and it follows that $N$ has radical length $q+1$.

We want to prove that the generating number of $k D_{4 q}$ does not exceed $q+1$. Note that when $q=1$, the dihedral group $D_{4}$ is just $C_{2} \times C_{2}$, and the claim follows from Theorem 4.9, so we assume that $q \geqslant 2$ from now on unless otherwise stated.

Now let $M$ be an indecomposable $k D_{4 q}$-module. By Theorem 4.19, it corresponds to a word satisfying one of the conditions (a), (b) or (c). Then $\operatorname{soc}(M)$ contains the submodule spanned by the vector spaces at positions of the form $b^{-1} a$ or $a^{-1} b$ (interpreted cyclically if $M$ is of the second kind). Such a position exists if $M$ is of the second kind since the condition that the word is not a power of a smaller word forces the word to contain both direct and inverse letters. However, such positions are removed in $M / \operatorname{soc}(M)$, so the indecomposable summands of $M / \operatorname{soc}(M)$ are of the first kind and correspond to words not containing $b^{-1} a$ or $a^{-1} b$.

Similarly, the indecomposable summands of $\operatorname{rad}(M)$ are of the first kind and correspond to words not containing $b a^{-1}$ or $a b^{-1}$. It follows that the indecomposable summands of $\operatorname{rad}(M / \operatorname{soc}(M))$ are of the first kind and correspond to words not containing $b^{-1} a, a^{-1} b, b a^{-1}$ or $a b^{-1}$. Thus the words must consist entirely of direct or inverse letters. But since $M(C) \cong M\left(C^{-1}\right)$, we can assume that the words only contain direct letters. By (a), the possible words are $(a b)^{q-1} a,(b a)^{q-1} b$, or sub-words of these. And we can prove

Lemma 4.21. Let $M$ be a $k D_{4 q}$-module of the first kind, with $q \geqslant 2$. If $M$ corresponds to a word that only contains direct letters, then its generating length is less than or equal to q.

Proof. We are going to show that

$$
\operatorname{gel}\left(M\left((a b)^{r} a\right)\right) \leqslant q \quad \text { and } \operatorname{gel}\left(M\left((a b)^{r}\right)\right) \leqslant q
$$

for $0 \leqslant r \leqslant q-1$, the case of words starting with $b$ being similar.

Since $D_{4 q}$ is a 2-group, the generating length of a module is always no more than its radical length, hence its dimension. So, for any word $C$, gel $(M(C)) \leqslant \operatorname{dim} M(C)=|C|+1$, where $|C|$ denotes the number of letters in $C$. Thus we are done if $r \leqslant q / 2-1$.

To handle $r \geqslant q / 2$, we temporarily introduce the following notation for modules with symmetry under reflection when exchanging $X$ with $Y$. For a word $u$, write $u^{\prime}$ for the inverse word with all $a$ s and $b$ s exchanged, so for example $\left(a b^{-1} a b\right)^{\prime}=a^{-1} b^{-1} a b^{-1}$. Write $M^{\prime}(u)$ for $M\left(u u^{\prime}\right)$ and $M^{\prime}(u, \phi)$ for $M\left(u u^{\prime}, \phi\right)$. Then $k D_{4 q}=M^{\prime}\left((a b)^{q}, i d\right)$, and one can see that $\tilde{\Omega} k=M^{\prime}\left(\left(b^{-1} a^{-1}\right)^{q-1} b^{-1}\right)$ and $\tilde{\Omega}^{-2} k=M^{\prime}\left((a b)^{q-1} a b^{-1}\right)$. It follows that we have short exact sequences

$$
0 \rightarrow k \rightarrow \tilde{\Omega}^{-2} k \rightarrow M\left((a b)^{q-1} a\right) \oplus M\left((b a)^{q-1} b\right) \rightarrow 0
$$

and

$$
0 \rightarrow k \rightarrow \tilde{\Omega} k \rightarrow M\left((a b)^{q-1}\right) \oplus M\left((b a)^{q-1}\right) \rightarrow 0 .
$$

Since $q \geqslant 2$, one sees that $\operatorname{gel}\left(M\left((a b)^{q-1} a\right)\right)=\operatorname{gel}\left(M\left((a b)^{q-1}\right)\right) \leqslant 2$, which handles the case $r=q-1$.

Now for $r \leqslant q-2, M\left((a b)^{r} a\right)$ and $M\left((a b)^{r}\right)$ embed in $M\left((a b)^{q-1}\right)$. Thus their ghost lengths are no more than the codimension plus two, and one can check that this is no more than $q$ when $r \geqslant q / 2$. 
In general, for a $p$-group $G$ and a $k G$-module $M$, we know that $M / \operatorname{rad}(M)$ and $\operatorname{soc}(M)$ are sums of trivial modules. Thus $\operatorname{rad}(M)$ is the fibre of a map $M \rightarrow \oplus k$ and $M / \operatorname{soc}(M)$ is the cofibre of a map $\oplus k \rightarrow M$. So

$$
\operatorname{gel}(M) \leqslant \operatorname{gel}(\operatorname{rad}(M))+1 \quad \text { and } \quad \operatorname{gel}(M) \leqslant \operatorname{gel}(M / \operatorname{soc}(M))+1 .
$$

Hence

$$
\operatorname{gel}(M) \leqslant \operatorname{gel}(\operatorname{rad}(M / \operatorname{soc}(M)))+2,
$$

and so by Lemma 4.21 and the discussion preceding it, the generating number of $k D_{4 q}$ does not exceed $q+2$. This is one more than the correct answer. We will show in Proposition 4.26 that the module $M\left((a b)^{\frac{q}{2}-1} a\right)$ has length $q$, so we can't improve this bound by improving Lemma 1.21 .

We will have to be a bit more clever in the construction to get the exact generating number. The above process takes two steps to produce a module $\operatorname{rad}(M / \operatorname{soc}(M))$ whose summands involve only direct letters, by removing "top" and "bottom" elements. We next show that we can add top elements instead of removing them, with the same effect, and as a result we will be able to do both steps at the same time.

Lemma 4.22. Let $M$ be a non-projective indecomposable module, with corresponding word $C$. There exists a short exact sequence

$$
0 \rightarrow M \rightarrow M^{\prime} \rightarrow \oplus k \rightarrow 0,
$$

where the indecomposable summands of $M^{\prime}$ are of the first kind and correspond to words that contain no $a b^{-1}$ or $b a^{-1}$.

Proof. First suppose that $M$ is of the first kind. If $C$ contains no $a b^{-1}$ or $b a^{-1}$, we simply set $M^{\prime}$ to be $M$. Otherwise, assume for example that $C$ contains $a b^{-1}$ and factor the word $C$ as $L_{1} L_{2}$, with $L_{1}$ ending with $a$ and $L_{2}$ starting with $b^{-1}$. Write $z$ for the basis element of $M(C)$ corresponding to the vertex connecting $L_{1}$ with $L_{2}$, and write $z_{i}$ for the corresponding basis element in $M\left(L_{i}\right)$, $i=1,2$. Then we have a short exact sequence $M \rightarrow M\left(L_{1}\right) \oplus M\left(L_{2}\right) \rightarrow k$, where the first map takes $z$ to $z_{1}-z_{2}$ and does the natural thing on the other basis elements, and the second map takes $z_{1}$ and $z_{2}$ to 1 in $k$ and the other basis elements to 0 . More generally, we can write $C=L_{1} L_{2} \cdots L_{n}$, broken at the spots $a^{-1} b$ and $b^{-1} a$, and set $M^{\prime}=\oplus M\left(L_{i}\right)$.

Now suppose that $M=M(C, \phi)$ is of the second kind, where $\phi: V \rightarrow V$ is an indecomposable automorphism. We can assume that $C=a^{-1} L b$ up to inverse and cyclic permutation. Fix a basis $v_{1}, \ldots, v_{n}$ of $V$, where $n=\operatorname{dim}(V)$. Let $M^{\prime \prime}=\oplus_{i=1}^{n} M_{i}$, with each $M_{i}=M(C)$. We write $w_{i}$ and $z_{i}$ for the basis elements in $M_{i}$ corresponding to the beginning and end of the word $C$. Then we have a short exact sequence $M(C, \phi) \rightarrow M^{\prime \prime} \rightarrow V$, where the first map sends $v_{i}$ to $\phi\left(w_{i}\right)-z_{i}$ for the first vertex and does the natural thing on the other vertices, and the second map sends $w_{i}$ to $v_{i}, z_{i}$ to $\phi\left(v_{i}\right)$ and the other basis elements to 0 . Here we regard $V$ as a module with trivial action. Since $M^{\prime \prime}$ is a sum of modules of the first kind, we can apply the process in the preceding paragraph to each summand to obtain a short exact sequence $M^{\prime \prime} \rightarrow M^{\prime} \rightarrow \oplus k$, with $M^{\prime}$ of the required form. It is not hard to see that the cokernel of the composite $M \rightarrow M^{\prime \prime} \rightarrow M^{\prime}$ also has a trivial action, and we are done.

Note that the short exact sequence is represented by a map $\oplus \Omega k \rightarrow M$, and this makes it possible to combine it with a map $\oplus k \rightarrow M$.

Example 4.23. We illustrate an example for $q=2$. Write $k V$ for the module $M\left(a^{-1} b^{-1} a b, i d_{k}\right)$ :

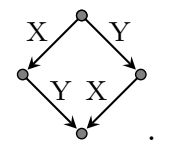

We begin by defining a cofibre sequence

$$
\Omega k \rightarrow k V \rightarrow M\left(a^{-1} b^{-1} a b\right) \rightarrow k .
$$

To see what the maps are, first consider the module 


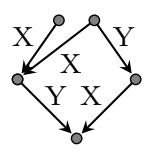

which has $k V$ as a codimension 1 submodule. We can choose a basis so that this becomes $M^{\prime}=$ $M\left(a^{-1} b^{-1} a b\right)$

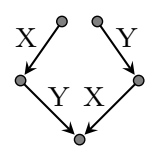

and the map $M^{\prime} \rightarrow k$ takes both top points to $k$ and has kernel $k V$. Then $M^{\prime}$ corresponds to a word that does not contain $b a^{-1}$ or $a b^{-1}$, and the summands of $M^{\prime} / \operatorname{soc}\left(M^{\prime}\right) \cong M(a) \oplus M(b)$ correspond to words that only contain direct letters. Note that the map from $k$ to $\operatorname{soc}\left(M^{\prime}\right)$ factors through $k V \rightarrow M^{\prime}$, so we can combine the two steps to get a cofibre sequence

$$
\Omega k \oplus k \rightarrow k V \rightarrow M(a) \oplus M(b) \rightarrow k \oplus \Sigma k .
$$

By Lemma 4.21, the generating length of the third term is at most $q$, which is 2 in our case.

Now we are ready to prove

Theorem 4.24. Let $k$ be a field of characteristic 2 . Then the generating number of $k D_{4 q}$ is at most $q+1$, for all $q \geqslant 1$.

Proof. The case when $q=1$ is dealt with in Theorem 4.9, so we prove the theorem for $q \geqslant 2$.

Let $M$ be a non-projective indecomposable module, with corresponding word $C$. In the short exact sequence $M \rightarrow M^{\prime} \rightarrow \oplus k$ from Lemma 4.22, the indecomposable summands of $M^{\prime}$ correspond to words that contain no $a b^{-1}$ or $b a^{-1}$. Hence the indecomposable summands of $M^{\prime \prime}=M^{\prime} / \operatorname{soc}\left(M^{\prime}\right)$ correspond to words of direct letters, and $\operatorname{gel}\left(M^{\prime \prime}\right) \leqslant q$.

We can form the octahedron

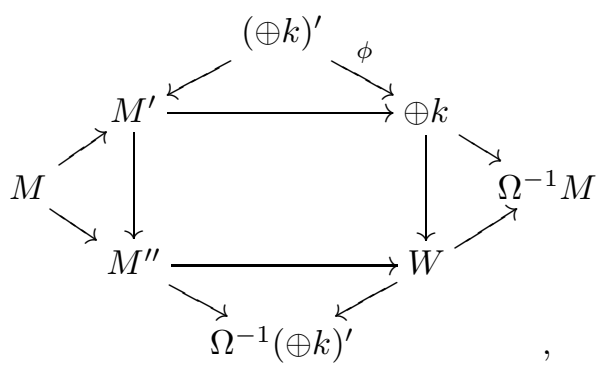

where $(\oplus k)^{\prime}$ is $\operatorname{soc}\left(M^{\prime}\right)$.

The proof will be finished once we show that $\operatorname{gel}(W)=1$. Here $W$ is the cofibre of a map $\phi$ between direct sums of trivial modules. Such a map is the sum of an identity map and a zero map. Hence $W$ is a direct sum of trivial modules $k$ and the modules $\Omega^{-1} k$, so gel $(W)=1$.

Corollary 4.25. Let $k$ be a field of characteristic 2. Then the ghost number and generating number of $k D_{4 q}$ are $q+1$ for all $q \geqslant 1$.

We now summarize and generalize the idea in the proof of the Theorem. Suppose that we start building an object $Q$ from $P, Y$ and $Z$ by first using a triangle

$$
P \rightarrow X \rightarrow Y \rightarrow \Sigma P
$$

and then using a triangle

$$
Q \rightarrow X \rightarrow Z \rightarrow \Sigma Q
$$


Then we can form the octahedron

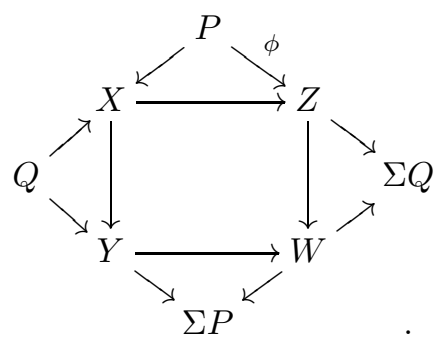

Assume that $P$ has length $m, Y$ has length $n$, and $Z$ has length $l$. Then the length of $Q$ does not exceed $m+n+l$. Indeed, $n+\operatorname{len}(W)$ bounds the length of $Q$. For example, if $\phi$ is in $\mathcal{I}^{s}$ for some positive integer, we have len $(W) \leqslant m+l-s$ by Lemma 3.1. Or, if $\phi=0$, then $W \cong Z \oplus \Sigma P$ and the two steps can be combined. This is analogous to the fact in topology that when a second cell is attached to a $C W$-complex without touching a first cell, then they can be attached to the complex at the same time.

We finish this section by computing the generating lengths of $M\left((a b)^{r}\right)$ and $M\left((a b)^{r} a\right)$, with $r \leqslant q / 2-1$. Note that there is a category automorphism on $\operatorname{StMod}\left(k D_{4 q}\right)$ induced by the group automorphism on $D_{4 q}$ that exchanges $x$ and $y$. It exchanges the $a$ 's and $b$ 's in the word which an indecomposable module corresponds to and preserves the ghost projective class. As a result,

$$
\operatorname{gel}\left(M\left((a b)^{r}\right)\right)=\operatorname{gel}\left(M\left((b a)^{r}\right)\right) \text { and } \operatorname{gel}\left(M\left((a b)^{r} a\right)\right)=\operatorname{gel}\left(M\left((b a)^{r} b\right)\right)
$$

for $D_{4 q}$-modules with $0 \leqslant r \leqslant q-1$.

Recall from Corollary 4.18 that the module $M=k D_{2 q}$ in $\operatorname{StMod}\left(k D_{4 q}\right)$ has its generating length equal to its radical length $q+1$. By Proposition 4.6, $\operatorname{gel}(\operatorname{rad}(M / \operatorname{soc}(M)))=\operatorname{gel}(M)-2=q-1$. Note that $M=M\left((a b)^{l+1}\left(a^{-1} b^{-1}\right)^{l+1}, i d\right)$, where $l=q / 2-1$, so $\operatorname{rad}(M / \operatorname{soc}(M)) \cong M\left((a b)^{l}\right) \oplus M\left((b a)^{l}\right)$. Then, since exchanging $a$ 's and $b$ 's preserves the generating length,

$$
\operatorname{gel}\left(M\left((a b)^{l}\right)\right)=\operatorname{gel}\left(M\left((b a)^{l}\right)\right)=q-1 .
$$

It follows that

$$
\operatorname{gel}\left(M\left((a b)^{r}\right)=2 r+1 \text { if } r \leqslant l,\right.
$$

and

$$
\operatorname{gel}\left(M\left((a b)^{r} a\right)=2(r+1) \text { if } r \leqslant l-1 .\right.
$$

We need to be a bit trickier to handle the module $M\left((a b)^{l} a\right)$.

Proposition 4.26. The $k D_{4 q}$-module $M\left((a b)^{l}\right.$ a) has generating length $q$, where $l=q / 2-1$.

Proof. We have a triangle

$$
\Sigma k \oplus k \rightarrow M \rightarrow M\left((a b)^{l} a\right) \oplus M\left((b a)^{l} b\right),
$$

where the map $\Sigma k \rightarrow M$ is a surjection.

Hence $\operatorname{gel}\left(M\left((a b)^{l} a\right) \oplus M\left((b a)^{l} b\right)\right) \geqslant q$. Since its radical length is $q$, this must be an equality. Then, using the symmetry again,

$$
\operatorname{gel}\left(M\left((a b)^{l} a\right)\right)=\operatorname{gel}\left(M\left((b a)^{l} b\right)\right)=q .
$$

\subsection{Ghost number of $C_{p^{r}} \times C_{p^{s}}$}

Let $G=C_{p^{r}} \times C_{p^{s}}$. In this section we show that

$$
\text { the ghost number of } k G \leqslant \text { the generating number of } k G \leqslant p^{r}+p^{s}-3
$$

and give the exact result when $p^{r}$ is 3 or 4 . Note that a general upper bound for the generating number for a $p$-group is given by the radical length of $k G$ minus 1 (Theorem 4.1). This gives $p^{r}+p^{s}-2$ for the group $C_{p^{r}} \times C_{p^{s}}$, and our result refines this upper bound by 1 . To keep the 
indices simple, we give a detailed proof for the group $C_{3} \times C_{3}$ at the prime 3 , and we indicate how to modify the proof to cover the general case. We are going to show that the composite of any three ghosts is stably trivial for the group $C_{3} \times C_{3}$, using Theorem 4.19.

Here is an overview of our strategy. Given a finitely generated projective-free module $N$ with radical length $n$ and an $l$-fold ghost $g: N \rightarrow N_{1}$ in $\operatorname{Mod}(k G)$, where $N_{1}$ is an arbitrary projective-free module, we can form the following commutative diagram:

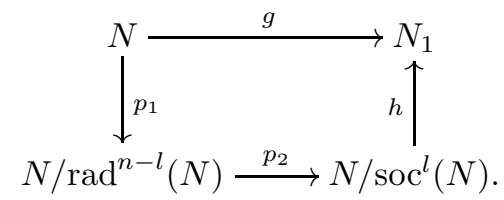

The $l$-fold ghost $g$ factors through $N / \operatorname{soc}^{l}(N)$ by Corollary 4.3, and the canonical projection $N \rightarrow$ $N / \operatorname{soc}^{l}(N)$ factors through $N / \operatorname{rad}^{n-l}(N)$ because $\operatorname{rad}^{n-l}(N) \subseteq \operatorname{soc}^{l}(N)$. If we have good control over the modules $N / \mathrm{rad}^{n-l}(N)$ or $N / \operatorname{soc}^{l}(N)$, we can factorize a long composite of ghosts as an $l$-fold ghost $g: N \rightarrow N_{1}$ followed by another composite of ghosts $f: N_{1} \rightarrow N_{2}$, and check whether $f$ is stably trivial on $N / \mathrm{rad}^{n-l}(N)$ or $N / \operatorname{soc}^{l}(N)$. For example, we can take $l$ to be $n-1$, so that $N / \operatorname{rad}(N)$ is a sum of trivial modules. Hence, if the map $f$ is a ghost, the composite $f \circ g$ is stably trivial, and so we have reproved that the generating length of $N$ is at most its radical length $n$ (Theorem 4.1). If we want to improve the bound, we need to choose $l$ smaller. We will take $l=n-2$.

While we can't classify $k\left(C_{p^{r}} \times C_{p^{s}}\right)$-modules, we can use Theorem 4.19 to classify certain quotient modules. We use that there is an isomorphism $k\left(C_{p^{r}} \times C_{p^{s}}\right) \cong k[X, Y] /\left(X^{p^{r}}, Y^{p^{s}}\right)$, where $X=x-1$ and $Y=y-1$, and $x$ and $y$ are the generators of the cyclic summands. Under this isomorphism, $\operatorname{rad}\left(k\left(C_{p^{r}} \times C_{p^{s}}\right)\right) \cong(X, Y)$ and $\operatorname{rad}^{2}\left(k\left(C_{p^{r}} \times C_{p^{s}}\right)\right) \cong\left(X^{2}, X Y, Y^{2}\right)$. Therefore $k\left(C_{p^{r}} \times C_{p^{s}}\right) / \operatorname{rad}^{2}\left(k\left(C_{p^{r}} \times C_{p^{s}}\right)\right) \cong \Lambda^{\prime}$, where $\Lambda^{\prime}=\Lambda /(X Y, Y X) \cong k[X, Y] /\left(X^{2}, Y^{2}, X Y\right)$ and $\Lambda=k\langle X, Y\rangle /\left(X^{2}, Y^{2}\right)$ is the ring from Section 4.6. Thus when $M$ is a $k\left(C_{p^{r}} \times C_{p^{s}}\right)$-module, $M / \operatorname{rad}^{2}(M)$ will be a $\Lambda^{\prime}$-module. Up to isomorphism, the indecomposable $\Lambda^{\prime}$-modules biject with the $\Lambda$-modules of Theorem 4.19 satisfying conditions (a) or (b) for $q=1$. Condition (c) is excluded by the requirement that $X Y$ be in the kernel.

Our proof will use this classification, so we will make it more explicit. A module satisfying condition (a) is of the first kind. If it has odd dimension, it is either the trivial module $k$; the module $M\left(\left(b^{-1} a\right)^{n}\right)$ for some positive integer $n$, which we say has shape " $W$ "; or the module $M\left(\left(a b^{-1}\right)^{n}\right)$ for some positive integer $n$, which we say has shape " $M$ ". For example, the " $M$ " module $M\left(\left(a b^{-1}\right)^{3}\right)$ looks like

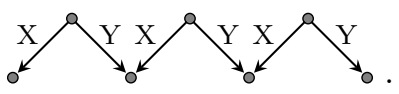

A module of the first kind with even dimension is one of the above with one end removed.

One can check that a module satisfying condition (b) of Theorem 4.19 corresponds to the word $b^{-1} a$, up to inverse and cyclic permutation. Recall that the additional data one needs to specify are a vector space $V$ with an indecomposable automorphism $\phi$. Since $\phi$ is indecomposable, one can choose a basis $\left\{v_{1}, v_{2}, \ldots, v_{m}\right\}$ for $V$ such that $\phi\left(v_{i}\right)=v_{i+1}$ for $i<m$. Thus we can view such a module as a quotient of an " $M$ " module, with a relation that identifies the right bottom basis element with a linear combination of the other bottom basis elements, as specified by $\phi\left(v_{m}\right)$.

We point out that this is very similar to the classification of $k V$-modules given in 2, Theorem 4.3.3], where $k$ has characteristic 2 .

Recall that the radical length of $k\left(C_{p^{r}} \times C_{p^{s}}\right)$ is $p^{r}+p^{s}-1$. If $N$ is projective-free, then its radical length $n$ is at most $p^{r}+p^{s}-2$, so we pick $l=p^{r}+p^{s}-4$. Note that $N / \operatorname{rad}^{2}(N)$ and $N / \operatorname{soc}^{l}(N)$ are naturally $\Lambda^{\prime}$-modules. And we have the following lemma, which helps describe summands of $N / \operatorname{soc}^{l}(N)$. 
Lemma 4.27. Let $G=C_{p^{r}} \times C_{p^{s}}$ be an abelian p-group of rank 2 with generators $x$ and $y$, respectively, and let $k$ be a field of characteristic $p$. Write $X=x-1$ and $Y=y-1$ in $k G$, and let $l=p^{r}+p^{s}-4$. Suppose $M$ is a $k G$-module containing elements $z_{0}, z_{2}$, and $z_{4}$ such that $Y z_{0}-X z_{2}$ and $Y z_{2}-X z_{4}$ are in $\operatorname{soc}^{l}(M)$. If $p^{s} \geqslant 3$, then $X z_{0}$ and $X z_{2}$ are in $\operatorname{soc}^{l}(M)$. Similarly, if $p^{r} \geqslant 3$, then $Y z_{2}$ and $Y z_{4}$ are in $\operatorname{soc}^{l}(M)$.

Intuitively, this is saying that we cannot have a "W"-shape in the module $M / \operatorname{soc}^{l}(M)$. In particular, only $k, M\left(a b^{-1}\right)$ and $M\left(\left(a b^{-1}\right)^{2}\right)$ can appear as indecomposable summands of $M / \operatorname{soc}^{l}(M)$ if $M$ is projective-free and $p^{r}, p^{s} \geqslant 3$. Note that to exclude a module like $M(a)$, one takes $z_{2}=z_{4}=0$, so the "W" isn't visible in this case.

Proof. Assume that $p^{s} \geqslant 3$. To show that $X z_{0} \in \operatorname{soc}^{l}(M)$, we need to show that it is killed by $\operatorname{rad}^{l}(k G)$, which is generated by $X^{p^{r}-1} Y^{p^{s}-3}, X^{p^{r}-2} Y^{p^{r}-2}$ and $X^{p^{r}-3} Y^{p^{s}-1}$ (where the last one is omitted if $p^{r}=2$ ). We compute

$$
\begin{gathered}
X^{p^{r}-1} Y^{p^{s}-3} X z_{0}=X^{p^{r}} Y^{p^{s}-3} z_{0}=0, \\
X^{p^{r}-2} Y^{p^{r}-2} X z_{0}=X^{p^{r}-1} Y^{p^{s}-3} Y z_{0}=X^{p^{r}} Y^{p^{s}-3} z_{2}=0,
\end{gathered}
$$

and

$$
X^{p^{r}-3} Y^{p^{s}-1} X z_{0}=X^{p^{r}-2} Y^{p^{s}-2} Y z_{0}=X^{p^{r}-1} Y^{p^{s}-3} Y z_{2}=X^{p^{r}} Y^{p^{s}-3} z_{4}=0,
$$

where we have made used of fact that $Y z_{0}-X z_{2}$ and $Y z_{2}-X z_{4}$ are killed by the generators. Hence $X z_{0} \in \operatorname{soc}^{l}(M)$. Similarly,

$$
\begin{gathered}
X^{p^{r}-1} Y^{p^{s}-3} X z_{2}=0 \\
X^{p^{r}-2} Y^{p^{s}-2} X z_{2}=X^{p^{r}-1} Y^{p^{s}-3} Y z_{2}=X^{p^{r}} Y^{p^{r}-3} z_{4}=0,
\end{gathered}
$$

and

$$
X^{p^{r}-3} Y^{p^{s}-1} X z_{2}=X^{p^{r}-3} Y^{p^{s}-1} Y z_{0}=0 .
$$

Hence $X z_{2} \in \operatorname{soc}^{l}(M)$. The other case is symmetrical.

We are now ready to prove the main theorem.

Theorem 4.28. Let $G=C_{3} \times C_{3}$, and let $k$ be a field of characteristic 3 . Then the ghost number of $k G$ is 3 .

Proof. As above, we write $x$ and $y$ for generators of the two factors of $C_{3} \times C_{3}$, and let $X=x-1$ and $Y=y-1$.

Theorem 4.9 gives a lower bound of 3 , so it suffices to show that the composite of any three ghosts in $\operatorname{Mod}(k G)$ out of a finitely-generated module is stably trivial. As we have explained, we consider the diagram

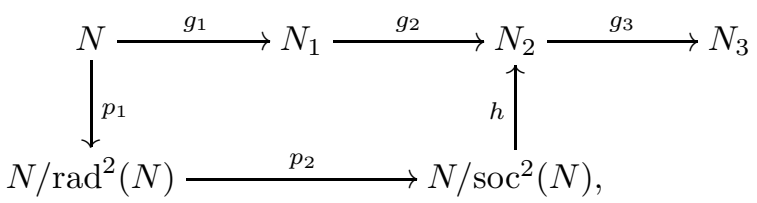

where $g_{1}, g_{2}$, and $g_{3}$ are ghosts in $\operatorname{Mod}(k G)$ and $N, N_{1}, N_{2}$, and $N_{3}$ are projective-free. Note that this diagram commutes in the module category. We will show that the composite $g_{3} \circ h \circ p_{2}$ is stably trivial, by restricting to each indecomposable summand $M$ of $N / \operatorname{rad}^{2}(N)$. We divide the summands $M$ into four cases, and write $j$ for the inclusion map $M \rightarrow N / \operatorname{rad}^{2}(N)$.

Case 1: $M$ is not of the form $k, M\left(a b^{-1}\right)$ or $M\left(\left(a b^{-1}\right)^{2}\right)$.

We claim that $\operatorname{soc}(M) \subseteq \operatorname{ker}\left(p_{2} \circ j\right)$, hence $p_{2} \circ j$ factors through a sum of trivial modules. Therefore, since $g_{3}$ is a ghost, the composite $g_{3} \circ h \circ p_{2} \circ j$ is stably trivial. We actually show that 
$p_{1}^{-1} j(\operatorname{soc}(M)) \subseteq \operatorname{soc}^{2}(N)$, which suffices, since $p_{2}$ kills $\operatorname{soc}^{2}(N)$. Observe using the classification that since $M$ is not $k, M\left(a b^{-1}\right)$ or $M\left(\left(a b^{-1}\right)^{2}\right)$, the elements $X\left(z_{0}\right), X\left(z_{2}\right), Y\left(z_{2}\right)$ and $Y\left(z_{4}\right) \operatorname{span} \operatorname{soc}(M)$ as $z_{0}, z_{2}$, and $z_{4}$ vary over elements satisfying $Y\left(z_{0}\right)=X\left(z_{2}\right)$ and $Y\left(z_{2}\right)=X\left(z_{4}\right)$. Suppose that we have $s \in p_{1}^{-1} j(\operatorname{soc}(M))$, say $p_{1}(s)=j\left(X\left(z_{0}\right)\right)$ for some $z_{0} \in M$ satisfying the above relations. Since $p_{1}$ is surjective, we have $\tilde{z_{0}}, \tilde{z_{2}}$, and $\tilde{z_{4}} \in N$ that project to $j\left(z_{0}\right), j\left(z_{2}\right)$, and $j\left(z_{4}\right)$, respectively. Then $p_{1}\left(Y\left(\tilde{z_{0}}\right)\right)=p_{1}\left(X\left(\tilde{z_{2}}\right)\right)$ and $p_{1}\left(Y\left(\tilde{z_{2}}\right)\right)=p_{1}\left(Y\left(\tilde{z_{4}}\right)\right)$. Since $N$ is projective-free, its radical length is at most 4 , hence $\operatorname{rad}^{2}(N) \subseteq \operatorname{soc}^{2}(N)$. Now we can apply Lemma 4.27 and see that $X\left(\tilde{z_{0}}\right) \in \operatorname{soc}^{2}(N)$. It follows that $s \in \operatorname{soc}^{2}(N)$ because $p_{1}(s)=p_{1}\left(X\left(\tilde{z_{0}}\right)\right)$. The other cases when $p_{1}(s)=j\left(X z_{2}\right), j\left(Y z_{2}\right)$, or $j\left(Y z_{4}\right)$ are similar.

Case 2: $M=M\left(a b^{-1}\right)$.

The map $p_{1}$ is surjective, so $g_{3} h p_{2}$ has its image in $\operatorname{rad}^{3}\left(N_{3}\right)$, using Corollary 4.3 and the fact that the diagram commutes in $\operatorname{Mod}(k G)$. $M$ has a basis $\{z, X z, Y z\}$ for some $z$ and the map $g_{3} h p_{2}$ sends $z$ to an element of the form $X^{2} Y w_{1}+X Y^{2} w_{2}$. After restriction to $M, g_{3} h p_{2}$ factors through the injective module which is free on two generators $v_{1}$ and $v_{2}$ via the maps sending $z$ to $X^{2} Y v_{1}+X Y^{2} v_{2}$, $v_{1}$ to $w_{1}$ and $v_{2}$ to $w_{2}$. Thus $g_{3} h p_{2}$ is stably trivial on $M$.

Case 3: $M=M\left(\left(a b^{-1}\right)^{2}\right)$.

The module $M\left(\left(a b^{-1}\right)^{2}\right)$ has schema $k z_{0} \stackrel{X}{\longleftarrow} k z_{1} \stackrel{Y}{\rightarrow} k z_{2} \stackrel{X}{\longleftarrow} k z_{3} \stackrel{Y}{\rightarrow} k z_{4}$. By considering the injective hull of $M\left(\left(a b^{-1}\right)^{2}\right)$, which is free on three generators, we see that a map out of it is stably trivial if it sends $z_{1}$ to $X Y^{2} w_{1}+X^{2} Y w_{2}$ and $z_{3}$ to $X Y^{2} w_{2}+X^{2} Y w_{3}$ for some elements $w_{1}, w_{2}$, and $w_{3}$. This is equivalent to $z_{1}$ being sent to $X \alpha$ and $z_{3}$ being sent to $Y \alpha$ for some $\alpha$ in the $2^{\text {nd }}$ radical.

To prove that this is the case, we form the following diagram:

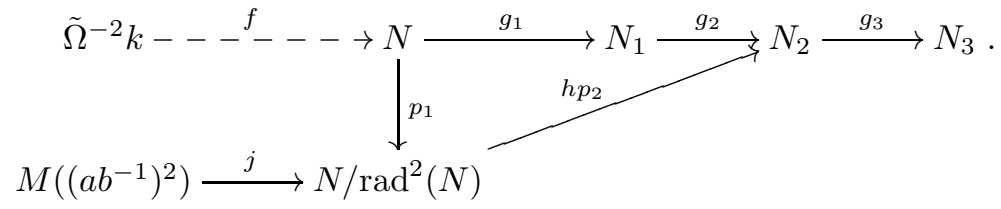

Writing $g=g_{3} \circ g_{2} \circ g_{1}$, we will show below that we can choose $\tilde{z}_{1}$ and $\tilde{z}_{3}$ in $N$ with

$$
g\left(\tilde{z}_{1}\right)=g_{3} h p_{2} j\left(z_{1}\right), \quad g\left(\tilde{z}_{3}\right)=g_{3} h p_{2} j\left(z_{3}\right), \quad \text { and } \quad Y \tilde{z}_{1}=X \tilde{z}_{3} .
$$

Since $\tilde{\Omega}^{-2} k$ is the free module on two generators $u_{1}$ and $u_{2}$ subject to the relation $Y u_{1}=X u_{2}$, the last displayed equality allows us to construct the dotted map $f$, by sending the generators to $\tilde{z}_{1}$ and $\tilde{z}_{3}$, respectively. We will now show that

$$
g\left(\tilde{z}_{1}\right)=X \alpha \quad \text { and } \quad g\left(\tilde{z}_{3}\right)=Y \alpha
$$

for some $\alpha \in \operatorname{rad}^{2}\left(N_{3}\right)$. Since $g_{1}$ is a ghost, the composite $g_{1} f$ is stably trivial. It follows that, modulo $\operatorname{soc}^{2}\left(N_{1}\right), g_{1}\left(\tilde{z}_{1}\right)=X \alpha^{\prime}$ and $g_{1}\left(\tilde{z}_{3}\right)=Y \alpha^{\prime}$ for some $\alpha^{\prime} \in N_{1}$. Since $g_{3} g_{2}$ is a double ghost, it kills $\operatorname{soc}^{2}\left(N_{1}\right)$ and takes $\alpha^{\prime}$ into $\operatorname{rad}^{2}\left(N_{3}\right)$. Hence we can set $\alpha=g_{3} g_{2}\left(\alpha^{\prime}\right)$.

We still need to pick the $\tilde{z}_{1}$ and $\tilde{z}_{3}$. First choose $\tilde{z}_{1}^{\prime}$ and $\tilde{z}_{3}^{\prime}$ in $N$ that project to $j\left(z_{1}\right)$ and $j\left(z_{3}\right)$ in $M\left(\left(a b^{-1}\right)^{2}\right)$, respectively. The difference $Y \tilde{z}_{1}^{\prime}-X \tilde{z}_{3}^{\prime}$ is in $\operatorname{rad}^{2}(N)$, say $Y \tilde{z}_{1}^{\prime}-X \tilde{z}_{3}^{\prime}=Y \beta-X \gamma$ for some $\beta$ and $\gamma \in \operatorname{rad}(N)$. We set $\tilde{z}_{1}=\tilde{z}_{1}^{\prime}-\beta$ and $\tilde{z}_{3}=\tilde{z}_{3}^{\prime}-\gamma$ so that $Y \tilde{z}_{1}=X \tilde{z}_{3}$. By Corollary 4.3, $g(\beta)=g(\gamma)=0$, hence

$$
g\left(\tilde{z}_{1}\right)=g\left(\tilde{z}_{1}^{\prime}\right)=g_{3} h p_{2} j\left(z_{1}\right) \text { and } g\left(\tilde{z}_{3}\right)=g\left(\tilde{z}_{3}^{\prime}\right)=g_{3} h p_{2} j\left(z_{3}\right) .
$$

Case 4: $M=k$, the trivial module.

Then clearly $g_{3} \circ h \circ p_{2}$ is stably trivial when restricted to $M$, since $g_{3}$ is a ghost.

Since we don't require the modules $N_{1}, N_{2}$, and $N_{3}$ to be finitely-generated in the proof, we have actually proved a stronger result, a bound for the generating number, giving:

Corollary 4.29. Let $k$ be a field of characteristic 3 . Then the generating number of $k\left(C_{3} \times C_{3}\right)$ is 3 . 
Remark 4.30. The arguments in this section go through for the group $G=C_{p^{r}} \times C_{p^{s}}$ with $2<p^{r} \leqslant$ $p^{s}$, and we get that the generating number of $k G$ is less than or equal to $p^{r}+p^{s}-3$. Theorem 4.9 gives a lower bound of $\left\lceil\frac{p^{r}-1}{2}\right\rceil+p^{s}-1$. In particular, if $p^{r}=3$, the ghost number of $k G$ is $p^{s}$, and if $p^{r}=4$, the ghost number of $k G$ is $p^{s}+1$.

We now indicate the modifications needed in the proof of the general case. Instead of $g_{2}$ being a ghost, we take it to be a $\left(p^{r}+p^{s}-5\right)$-fold ghost. Then the map $h$ has domain $N / \operatorname{soc}^{p^{r}+p^{s}-4}(N)$. In Case 1, one checks that $p_{1}^{-1} j(\operatorname{soc}(M)) \subseteq \operatorname{soc}^{p^{r}+p^{s}-4}(N)$. In Case 2, the map $g_{3} h p_{2}$ sends $z \in$ $M\left(a b^{-1}\right)$ to an element of the form $X^{p^{r}-1} Y^{p^{s}-2} w_{1}+X^{p^{r}-2} Y^{p^{s}-1} w_{2}$. In Case 3, a map out of $M\left(\left(a b^{-1}\right)^{2}\right)$ is stably trivial if it sends $z_{1}$ to $X \alpha$ and $z_{3}$ to $Y \alpha$ for some $\alpha$ in the $\left(p^{r}+p^{s}-4\right)^{t h}$ radical. Case 4 is unchanged.

\subsection{Possible ghost numbers for group algebras}

In this section, we classify group algebras with certain small ghost numbers, and also put constraints on which ghost numbers can occur. Whenever we write $k G, k$ can be any field whose characteristic divides the order of $G$.

In [9] it is shown that the abelian groups $G$ such that the ghost number of $k G$ is 2 are $C_{4}, C_{2} \times C_{2}$ and $C_{5}$. The results of the previous section and Theorem 4.9 give a complete list of abelian $p$-groups of ghost number 3:

Proposition 4.31. Let $G$ be an abelian p-group. Then the ghost number of $k G$ is 3 if and only if $G$ is $C_{7}, C_{3} \times C_{3}$, or $C_{2} \times C_{2} \times C_{2}$ if and only if the generating number of $k G$ is 3 .

Below we will extend this to non-abelian $p$-groups, with one ambiguous group. We first recall a consequence of Jennings' formula which will also be useful in studying the gaps in the possible ghost numbers.

Lemma 4.32 (2, Thm. 3.14.6]). Let $k$ be a field of characteristic $p$. If $G$ is a group of order $p^{r}$, then

nilpotency index of $J\left(k\left(C_{p}^{r}\right)\right) \leqslant$ nilpotency index of $J(k G) \leqslant$ nilpotency index of $J\left(k\left(C_{p^{r}}\right)\right)$.

Note that the nilpotency index of $J\left(k\left(C_{p}^{r}\right)\right)$ is $r(p-1)+1$.

Proposition 4.33. Let $k$ be a field of characteristic $p$. If $G$ is a group of order $p^{r}$, then the ghost number of $k G$ is at least $(r-1)(p-1)+1$.

Proof. The group $G$ has a quotient $H$ of order $p^{r-1}$. By Theorem 4.15, radlen $(k H)$ is a lower bound for the ghost number of $k G$. Now by the previous lemma, radlen $(k H) \geqslant(r-1)(p-1)+1$, so we are done.

Theorem 4.34. The following is a complete list of the p-groups $G$ such that $k G$ has the specified ghost number:

1: the abelian groups $C_{2}$ and $C_{3}$;

2: the abelian groups $C_{4}, C_{2} \times C_{2}$ and $C_{5}$;

3: the abelian groups $C_{7}, C_{3} \times C_{3}$ and $C_{2} \times C_{2} \times C_{2}$, the dihedral group $D_{8}$ of order 8 , and possibly the quaternion group $Q_{8}$, which has ghost number 3 or 4.

In each case, except possibly for $Q_{8}$, the generating number equals the ghost number.

Proof. The case of ghost number 1 is the main result of [3].

A non-abelian $p$-group must have order $p^{r}$ for $r \geqslant 3$, so by Proposition 4.33 it must have ghost number at least 3 . Thus a $p$-group of ghost number 2 must be abelian, and this case is proved in [9].

The only ways for $(r-1)(p-1)+1$ to equal 3 are $p^{r}=8$ or 9 . The non-abelian groups of order 8 are $D_{8}$ and $Q_{8}$, which are discussed in Corollary 4.25, Theorem 4.28 and Corollary 4.29, and there are no non-abelian groups of order 9. The abelian case is Proposition 4.31. 
Next we observe that, for a fixed prime $p$, not all positive integers can be the ghost number of some $p$-group. For example, since the generating hypothesis fails for $p>3$, the number 1 cannot be the ghost number of a $p$-group with $p>3$. On the other hand, the elementary abelian 2 -group of rank $l$ has ghost number $l-1$, so every positive integer can be a ghost number at the prime 2 . Here is a result giving gaps in the possible ghost numbers at odd primes.

Theorem 4.35. Let $p$ be an odd prime, and let $k$ be a field of characteristic $p$. Write $\left(l_{1}, l_{2}, l_{3}, \ldots\right)$ for the increasing sequence of integers that are ghost numbers of the group algebras $k G$, with $G$ being a p-group. Then $l_{1}=\frac{p-1}{2}$,

$$
\frac{3(p-1)}{2} \leqslant l_{2}=\text { ghost number of } C_{p} \times C_{p} \leqslant 2 p-3,
$$

and $\min \left(\frac{p^{2}-1}{2}, 2 p-1\right) \leqslant l_{3}$.

Proof. We know that the ghost number of $C_{p}$ is $\frac{p-1}{2}$ and that of $C_{p^{2}}$ is $\frac{p^{2}-1}{2}$ [9, Thm. 5.4]. And the ghost number of $C_{p} \times C_{p}$ is constrained by Theorem 4.9 and Remark 4.30 :

$$
\frac{3(p-1)}{2} \leqslant \text { ghost number of } C_{p} \times C_{p} \leqslant 2 p-3 .
$$

By Proposition 4.33, the groups of order $p^{r}$ with $r \geqslant 3$ have ghost numbers at least $2 p-1$. Comparing these numbers, we get

$$
\frac{p-1}{2} \leqslant 2 p-3 \leqslant \min \left(\frac{p^{2}-1}{2}, 2 p-1\right)
$$

and the theorem follows.

Thus one sees that for large primes there are large gaps in the sequence of possible ghost numbers. Observe that when $p \geqslant 5$,

$$
\text { the ghost number of } k\left(C_{p}^{3}\right) \leqslant 3 p-3 \leqslant \frac{p^{2}-1}{2}=\text { the ghost number of } k C_{p^{2}},
$$

where the first inequality uses Theorem 4.1. And by Theorem 4.9 and Proposition 4.33, the ghost number of $k\left(C_{p}^{r}\right)$ is no more than the ghost number of any p-group with larger size. We conjecture that this is also true for groups of the same size, which would imply that $l_{3}$ is the ghost number of $k\left(C_{p}^{3}\right)$ when $p \geqslant 5$. The following conjecture should be viewed as the stabilized version of Lemma 4.32 .

Conjecture 4.36. Let $k$ be a field of characteristic $p$. If $G$ is a p-group of order $p^{r}$, then ghost number of $k\left(C_{p}^{r}\right) \leqslant$ ghost number of $k G \leqslant$ ghost number of $k\left(C_{p^{r}}\right)$.

\section{REFERENCES}

[1] M. Auslander and I. Reiten. Representation theory of Artin algebras IV. Comm. in Algebra, 5(5) (1977), 443518.

[2] D. J. Benson. Representations and cohomology I. Cambridge Univ. Press, Cambridge, 1998.

[3] D. J. Benson, S. K. Chebolu, J. D. Christensen and J. Mináč. The generating hypothesis for the stable module category of a p-group. Journal of Algebra 310(1) (2007), 428-433.

[4] A. Bondal and M. Van den Bergh. Generators and representability of functors in commutative and noncommutative geometry. Moscow Math. J. 3 (2003), 1-36.

[5] A. M. Bohmann and J. P. May. A presheaf interpretation of the generalized Freyd conjecture. Theory Appl. Categ. 26(16) (2012), 403-411.

[6] J. F. Carlson. Modules and group algebras. Lectures in Mathematics, ETH Zürich. Birkhäuser Verlag, Basel, 1996. Notes by Ruedi Suter.

[7] J. F. Carlson, S. K. Chebolu and J. Mináč. Freyd's generating hypothesis with almost split sequences. Proc. Amer. Math. Soc. 137 (2009), 2575-2580.

[8] S. K. Chebolu, J. D. Christensen and J. Mináč. Groups which do not admit ghosts. Proc. Amer. Math. Soc. 136(4) (2008), 1171-1179. 
[9] S. K. Chebolu, J. D. Christensen and J. Mináč. Ghosts in modular representation theory. Advances in Mathematics 217(6) (2008), 2782-2799.

[10] S. K. Chebolu, J. D. Christensen and J. Mináč. Freyd's generating hypothesis for groups with periodic cohomology. Canadian Mathematical Bulletin, 55(1) (2012), 48-59.

[11] J. D. Christensen. Ideals in triangulated categories: phantoms, ghosts and skeleta. Advances in Mathematics 136(2) (1998), 284-339.

[12] J. D. Christensen and G. Wang. Ghost numbers of group algebras II. Preprint, arXiv:1310.5682, 2013.

[13] P. Freyd. Stable homotopy. In Proc. Conf. Categorical Algebra (La Jolla, Calif., 1965), pages 121-172. Springer, New York, 1966.

[14] S. A. Jennings. The structure of the group ring of a $p$-group over a modular field. Trans. Amer. Math. Soc. 50 (1941), 175-185.

[15] H. Krause. Auslander-Reiten theory via Brown representability. K-Theory 20(4) (2000), 331-344. 